\title{
Facile and Fast Transformation of Non-Luminescent to Highly Luminescent MOFs: Acetone Sensing for Diabetes Diagnosis and Lead Capture from Polluted Water
}

\author{
Mario Gutiérrez, ${ }^{a}$ Annika F. Möslein, ${ }^{a}$ and Jin-Chong Tan ${ }^{a *}$
}

${ }^{a}$ Multifunctional Materials \& Composites (MMC) Laboratory, Department of Engineering Science, University of Oxford, Parks Road, Oxford OX1 3PJ, United Kingdom.

\begin{abstract}
Metal-organic frameworks (MOFs) stand as one of the most promising materials for the development of advanced technologies owing to their unique combination of properties. The conventional synthesis of MOFs involves a direct reaction of the organic linkers and metal salts, however, their post-synthetic modification is a sophisticated route to produce new materials or to confer novel properties that cannot be attained through traditional methods. This work describes the post-synthetic MOF-to-MOF transformation of a non-luminescent MOF (Zn-based OX-1) into a highly luminescent framework (Ag-based OX-2) by a simple immersion of the former in a silver salt solution. The conversion mechanism exploits the uncoordinated oxygen atoms of terephthalate linkers found in $\mathrm{OX}-1$, instead of the unsaturated metal sites commonly employed, making the reaction much faster. The materials derived from the OX-1 to OX-2 transformation are highly luminescent and exhibit a selective response to acetone, turning them into a promising candidate
\end{abstract}


for manufacturing fluorometric sensors for the diagnosis and monitoring of diabetes mellitus. Our methodology can be extended to other metals such as lead $(\mathrm{Pb})$. The fabrication of a polymer mixed-matrix membrane containing $\mathrm{OX}-1$ is used as a proof-of-concept for capturing $\mathrm{Pb}$ ions (as pollutants) from water. This research instigates the exploration of alternative methodologies to confer MOFs with special aptitudes for photochemical sensing or for environmental applications such as water purification.

Keywords: MOF-to-MOF transformation, Luminescence, Acetone Sensing, Diabetes Monitoring, $\mathrm{Pb}$ capture, Water Purification.

\section{Introduction}

Metal-organic frameworks (MOFs) are a class of porous ordered structures constructed from a periodic self-assembly of organic and inorganic subunits. ${ }^{1}$ Over the last two decades, MOFs have received great attention from a broad interdisciplinary number of scientists, including chemists, physicists, material scientists or engineers among others. The versatile physicochemical properties of MOFs make them potential candidates for emerging key technologies. ${ }^{2-4}$

MOFs are typically synthesized by a direct reaction of the organic linkers and the metal salts through numerous synthetic approaches. ${ }^{5}$ However, during the past few years, distinct routes have been investigated in order to develop new MOF materials with striking properties that could not be achieved through conventional methods. One of the most deeply explored mechanism is the post-synthetic modification of MOFs. ${ }^{6-8}$ Although most of the examples were based on the modification of the organic linkers, ${ }^{9-11}$ an increasing number of cases employing metal exchange have been reported. ${ }^{12,} 13$ The post-synthetic modifications by leveraging the metal exchange approach can be accomplished through a charge balanced substitution of the cations present in the 
pores, or by a more sophisticated process which encompasses the rupture and formation of coordination bonds. ${ }^{14}$ Therefore, the cation exchange process will be more effective in those metals containing coordinatively unsaturated sites, or metals with saturated positions that can still undergo cation exchange as a result of weak field interactions with the organic linkers. ${ }^{13}$ The coexistence of multiple metals in a single MOF crystal generates a subclass of materials known as the mixedmetal MOFs (MM-MOFs). ${ }^{15}$ Some MM-MOFs have exhibited improved performance in catalysis, ${ }^{16,17}$ photocatalysis $^{18,19}$ and gas adsorption ${ }^{20,21}$ technologies, while others have also shown outstanding luminescent properties, making them promising candidates to be deployed in the fabrication of disruptive devices, such as luminescent thermometers, ${ }^{22,23}$ and optical sensors..$^{24}$, 25

The substitution of metal cations in MOFs can also induce a complete MOF-to-MOF transformation. For instance, HKUST-1 has been converted to MIL-100 by the immersion of the former in a $\mathrm{FeCl}_{3} \cdot 6 \mathrm{H}_{2} \mathrm{O}$ solution for several hours at room temperature. ${ }^{26}$ This is a clear example of a facile and mild transmetalation methodology to obtain MIL-100, which is conventionally produced by using harsh synthetic conditions (hydrofluoric acid, $150{ }^{\circ} \mathrm{C}, 6$ days). ${ }^{26}$ Another stimulating example is given by the transformation of the bio-MOF-1 to a Ag-based MOF by exposing the former to a silver nitrate solution. ${ }^{27}$ The transformed Ag-MOF displayed an intense light blue emission, not observed in the pristine bio-MOF-1, thus conferring the fascinating properties of this new material.

Inspired by the foregoing examples, we investigate the MOF-to-MOF transformation from a non-luminescent material to highly luminescent MOF systems. The transmetalation processes described above require the extended immersion of the MOF in the desired metal salt solution from several hours to weeks, making this methodology sluggish and less practical. 
Herein, we report a facile and fast methodology, taking advantage of the uncoordinated oxygen atoms of the terephthalate linkers present in the OX-1 MOF. ${ }^{28-30}$ These uncoordinated oxygen atoms will promptly interact with the $\mathrm{Ag}$ cations of a silver nitrate $\left(\mathrm{AgNO}_{3}\right)$ solution, leading to the transformation of OX-1 (Zn-BDC, where BDC = 1,4-benzene dicarboxylate) to OX-2 (AgBDC) MOF, termed as Ag/OX-1 materials. Remarkably, and unlike the pristine OX-1, the new $\mathrm{Ag} / \mathrm{OX}-1$ transformed materials exhibit an intense green luminescence with an emission quantum yield of up to $22 \%$ in the solid-state (powder) form. Additionally, this green emission is strongly quenched in the presence of acetone ( $>90 \%$ reduction in initial emission intensity), turning these materials into attractive candidates for the future fabrication of breathalyzers for diabetes mellitus detection and monitoring. ${ }^{31-33}$ This transformation mechanism was also extended to lead $(\mathrm{Pb})$ cations. As they are well-known toxic pollutants present in aquatic environments, ${ }^{34,}, 35$ a fast conversion of OX-1 to Pb-BDC MOFs could be an easy to implement low-cost route to capture $\mathrm{Pb}$ ions from polluted water. To this end, we dispersed the OX-1 MOF in a hydrophobic polyurethane (PU) to fabricate a mixed-matrix membrane, which was then tested by immersing it in a $\mathrm{Pb}\left(\mathrm{NO}_{3}\right)_{2}$ water solution. After just 20 minutes of immersion, we proved that $\mathrm{OX}-1$ is effectively transformed to a $\mathrm{Pb}-\mathrm{BDC} \mathrm{MOF}$, and therefore this proof-of-concept may be exploited for the depuration of aquatic environments.

\section{Results and Discussion}

\section{Fast Transformation of OX-1 to OX-2 in Methanol}

To begin with, we explore the post-synthetic MOF transformation by leveraging the uncoordinated oxygen atoms of the BDC linker found in the OX-1 MOF (Fig. 1A). The Ag cations of a solution of $\mathrm{AgNO}_{3}$ in methanol will promptly interact with the uncoordinated oxygen atoms, leading to the 
transformation of the non-luminescent OX-1 to the luminescent OX-2 MOF. To unveil the mechanism of the MOF transformation, the crystalline structure, morphology and spectroscopic properties were determined by means of powder X-ray diffraction (PXRD), Fourier transform infrared (FTIR) spectroscopy, scanning electron microscopy coupled to energy dispersive X-ray spectroscopy (FESEM-EDX), atomic force microscopy (AFM) coupled to local probe nanoFTIR, and steady-state fluorescence spectroscopy.

Upon the immersion of $250 \mathrm{mg}$ of OX-1 MOF in a solution of $120 \mathrm{mg}(0.7 \mathrm{mmol})$ of $\mathrm{AgNO}_{3}$ in $20 \mathrm{~mL}$ of $\mathrm{MeOH}$ (see experimental part), an instantaneous change from a non-luminescent to a highly luminescent material exhibiting a green emission was observed under UV (365 nm) lamp
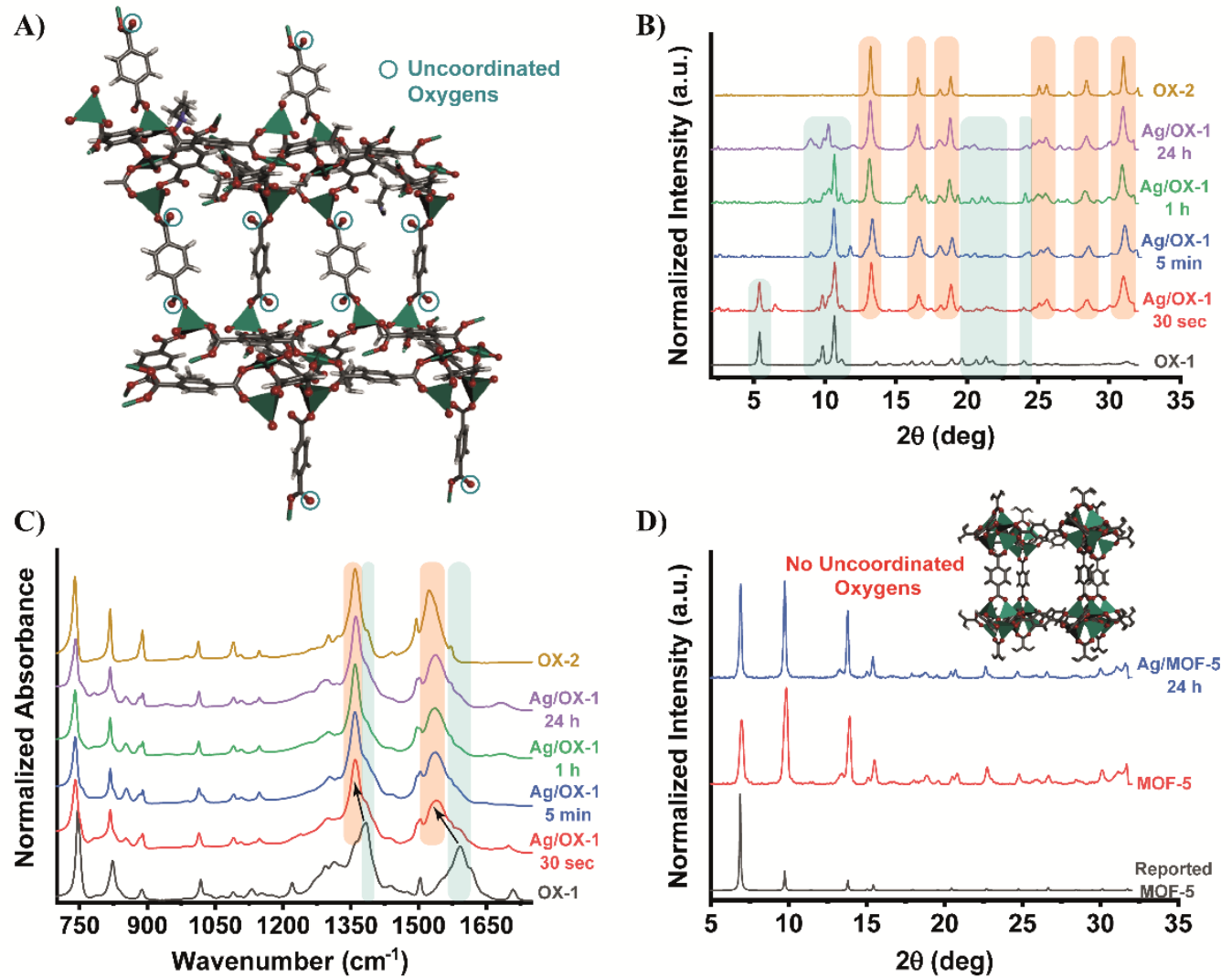

Figure 1. A) Representation of the 3D structure of OX-1 MOF, where the blue circles indicate the uncoordinated oxygen atoms. B) PXRD patterns and C) FTIR spectra of pristine OX-1 and OX-2 MOFs and the different converted Ag/OX-1 materials. D) PXRD patterns of the reported and as-synthesized MOF-5 and the nontransformed Ag/MOF-5 material. The inset is a representation of the 3D structure of MOF-5. 
irradiation (see video demo in Supporting Information, SI). Four different batches were prepared by interrupting the transformation reaction at different times ranging from $30 \mathrm{~s}$ to $24 \mathrm{hr}$, yielding the materials termed as Ag/OX-1 30s, Ag/OX-1 5min, Ag/OX-1 1 hr and Ag/OX-1 24 hr. Fig. 1B shows the PXRD patterns of the pristine OX-1 and OX-2 MOFs along with the transformed Ag/OX-1 materials. While the Bragg diffraction peaks of the pristine OX-1 and OX-2 resemble those previously reported, ${ }^{28,30,36} \mathrm{Ag} / \mathrm{OX}-1$ materials reveal a combination of diffraction peaks characteristic of the OX-1 and OX-2 MOFs, even after just $30 \mathrm{~s}$ of reaction (Fig. 1B). For the $\mathrm{Ag} / \mathrm{OX}-1$ materials converted at times longer than $30 \mathrm{~s}$, the Bragg peak characteristic of OX-1 at a $2 \theta$ angle of $\sim 5^{\circ}$ (plane (200)) completely vanishes, indicating that at least part of the OX-1 MOF crystals is transformed to OX-2. However, after $24 \mathrm{~h}$ of reaction, there are still some diffraction peaks typical of OX-1 (Fig. 1B), which leads to the conclusion that even after this period, there is a partial, but not complete MOF-to-MOF transformation. In the same line, the FTIR spectra provide more proof about the conversion (Fig. 1C). While most of the peaks at lower energies remain unaffected, as they are characteristic vibrations of the BDC linker, ${ }^{29,}, 37,38$ the peaks at $\sim 1600$ and $\sim 1390 \mathrm{~cm}^{-1}$, associated with the asymmetric and symmetric stretching vibrations of the carboxylic groups of the BDC linker coordinated to the metal centre ${ }^{29,} 39$ are strongly shifted to lower energies ( 1520 and $1360 \mathrm{~cm}^{-1}$, Fig. 1C). The observed shifts agree with a change in the coordination of the BDC linkers, which will be mostly linked to the Ag ions instead of the $\mathrm{Zn}$ ones. This interpretation is confirmed by the fact that the FTIR spectrum of pristine OX-2 MOF exhibits similar IR vibrations at 1520 and $1360 \mathrm{~cm}^{-1}$ assigned to the asymmetric and symmetric stretching vibrations of the carboxylic groups of the BDC linkers. ${ }^{40}$ However, the contribution of the bands at 1600 and $1390 \mathrm{~cm}^{-1}$ is still being detected (Fig. 1C), revealing that not all OX-1 crystals are fully converted to OX-2, as previously deduced from the XRD results. The rapid transformation 
of OX-1 to OX-2 supports our theory that the uncoordinated oxygen atoms of BDC linkers are responsible for this transmetalation reaction. To interrogate this effect further, we applied the same methodology but by replacing OX-1 with MOF-5, which is built using the same Zn cations and BDC linkers, however, the oxygen atoms in the latter framework are fully coordinated (Fig. 1D inset). ${ }^{41,42}$ The XRD pattern of the MOF-5 after being soaked for $24 \mathrm{hr}$ in a methanolic solution of $\mathrm{AgNO}_{3}$ is the same as the pristine MOF-5 (Fig. 1D). In addition, no green light emission was observed under UV irradiation. This result unequivocally proves that the transformation does not occur for MOF-5, evidencing that the reaction in OX-1 may involve the uncoordinated oxygen atoms in the linkers instead of the commonly described mechanism that exploits the uncoordinated metal sites. ${ }^{13}$

To unravel how the OX-1 conversion affects the morphology of the crystals, and to further answer the question about the metal composition of the $\mathrm{Ag} / \mathrm{OX}-1$ composite, we have performed the FESEM-EDX characterization (Fig. 2 and Fig. S1-4 in SI). Interestingly, the SEM micrographs of pristine OX-1 show a heterogeneous distribution of micron-sized crystals (Fig. S1A) as well as small elongated nanoplates (Fig. S1B). This heterogeneous distribution will be crucial, as the large surface area of the small nanoplates can facilitate the transformation process. This hypothesis is confirmed by carefully examining the FESEM micrographs and the corresponding EDX maps of the transformed Ag/OX-1 materials. Fig. 2 illustrates different regions of the Ag/OX-1 30 s. In these images, we pinpointed two distinctive regions: (i) the larger micro-sized crystals which are mainly composed of $\mathrm{Zn}$ metal (Fig. 2A-F), and (ii) relatively smaller nano-sized crystals, where only Ag traces can be determined (Fig. 2G-H). We can thus conclude that the large crystals of OX1 remain stable when immersed in the methanolic Ag solution, whereas the nanoplates - with their larger surface area - transform from $\mathrm{OX}-1$ to $\mathrm{OX}-2$, as the $\mathrm{Zn}$ ions are fully replaced by $\mathrm{Ag}$ ions. 
The coexistence of the larger crystals of OX-1 and the finer nanostructures of OX-2 is consistent with the results obtained from the PXRD and FTIR studies. FESEM micrographs and EDX maps of the transformed materials at different times of reactions (Ag/OX-1 $5 \mathrm{~min}, 1 \mathrm{hr}$ and $24 \mathrm{hr}$ ) also reveal the coexistence of the OX-1 large crystals and the OX-2 nanostructures (transformed from OX-1), as shown in Fig. S2-S4. To further validate these results, the crystals of different sizes have been investigated by AFM coupled with near-field infrared spectroscopy (nanoFTIR). We have recently demonstrated that this technique is a powerful tool to chemically characterize MOFs at a single crystal level with a resolution down to $20 \mathrm{~nm} .{ }^{43}$ Fig. 3 shows the AFM topography images belonging to the large (Fig. 3A) and small (Fig. 3B) crystals found in the sample after the
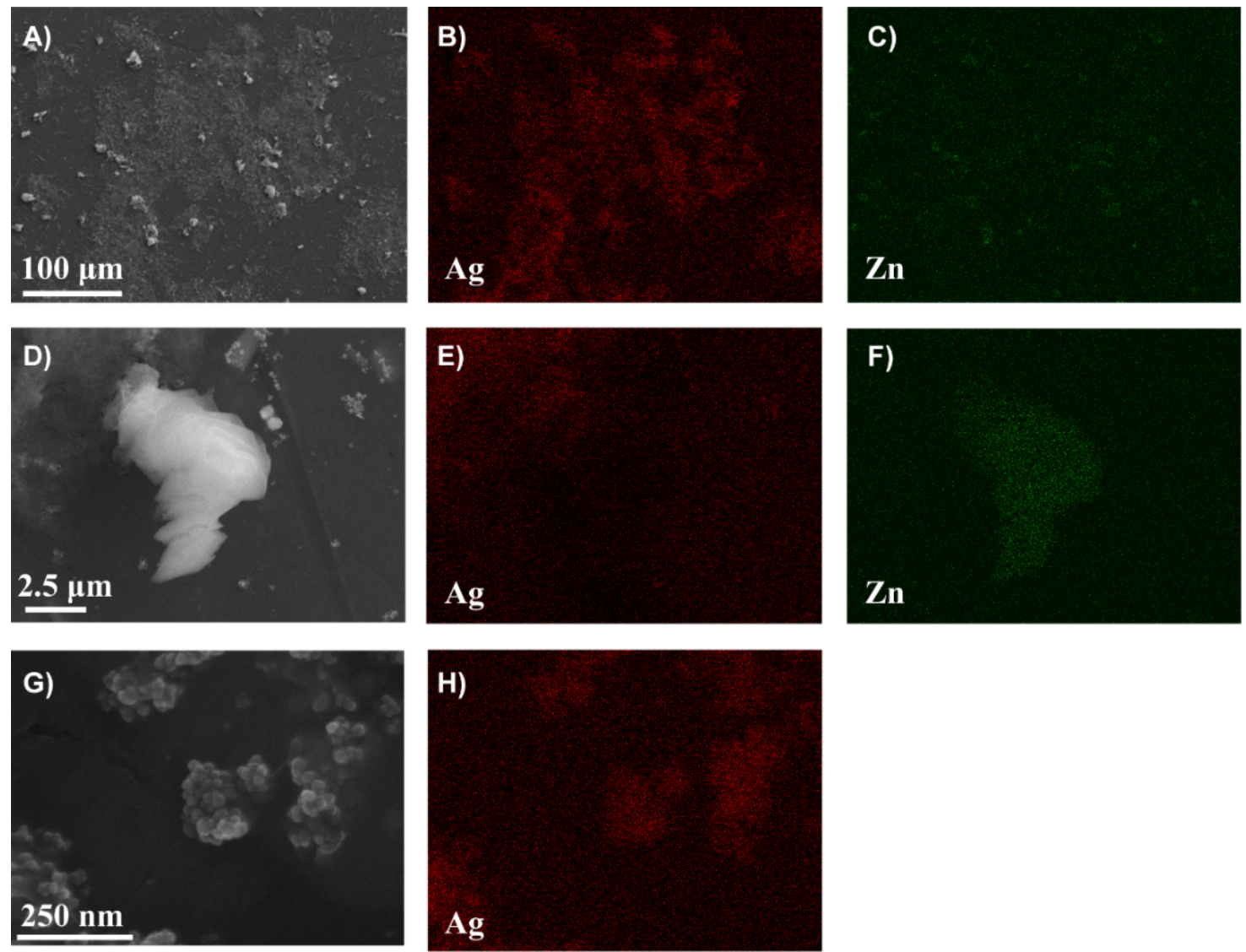

Figure 2. FESEM-EDX images of different regions found in Ag/OX-1 30 s. A-C) Display a large area where big OX-1 and small OX-2 crystals coexist. D-E) A larger crystal of OX-1 where only Zn atoms are detected, while GH) is a magnified region containing only OX-2 (Ag-based) small crystals. 
transformation process, together with the nanoFTIR spectra locally probed on each type of crystals (Fig. 3C). The nanoFTIR spectrum of the large crystals (region 1) resembles that of OX-1 with bands at $\sim 1600$ and $\sim 1390 \mathrm{~cm}^{-1}$, while the spectrum recorded on the small nanocrystals (region 2) is similar to that observed for OX-2 crystals with characteristics peaks at $\sim 1520$ and $\sim 1360 \mathrm{~cm}^{-1}$. These findings unambiguously prove that the larger micron-sized crystals correspond to the nontransformed OX-1 MOF, while the smaller nanocrystals are OX-2 MOF nanoplates transformed from OX-1.

The main objective behind this MOF-to-MOF transformation was to impart a fluorescent property on the new transformed MOFs, as we previously reported that OX-2 is highly luminescent. ${ }^{40}$ The transformed Ag/OX-1 (30 s, $5 \mathrm{~min}, 1 \mathrm{hr}$ and $24 \mathrm{hr}$ ) materials are all highly emissive with a
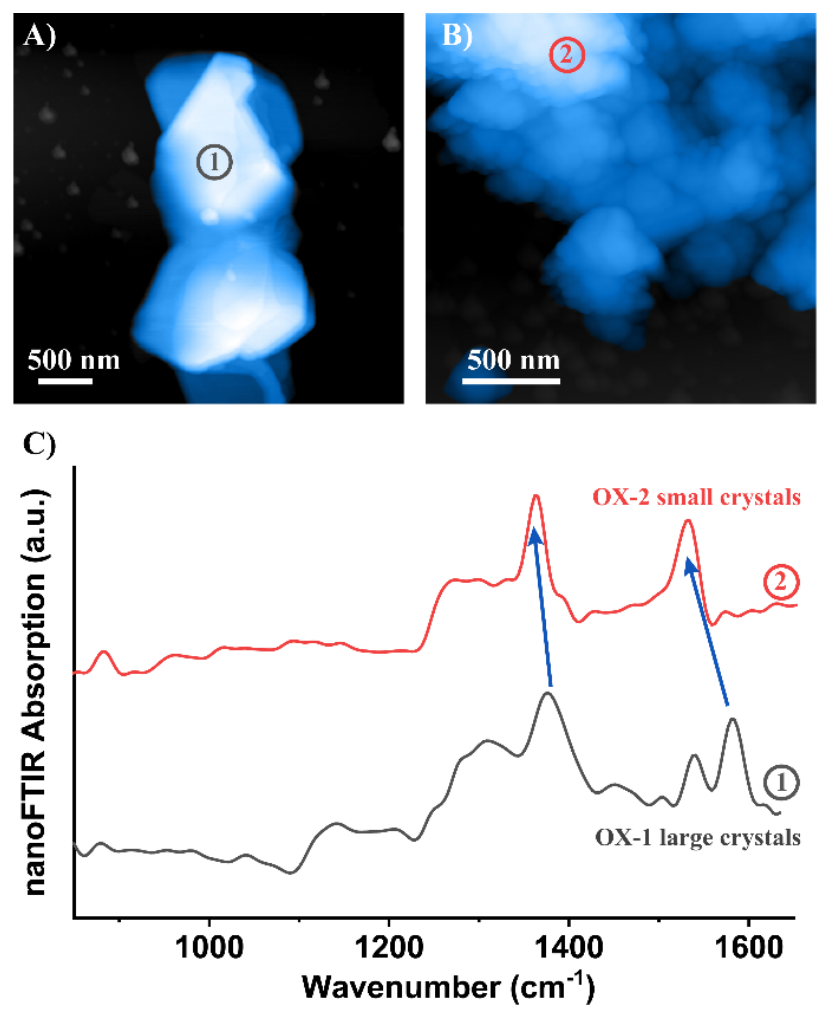

Figure 3. AFM topography images of Ag/OX-1 24h at selected regions: A) large crystals, and B) small nanocrystals. C) Local probe nanoFTIR spectra of Ag/OX-1 $24 \mathrm{~h}$ recorded at the regions shown in panels (A) and (B), respectively. 
fluorescence quantum yield of around $\sim 20 \%$ in the solid-state powder form (Fig. 4A,C and S5). The emission spectra resemble that of pristine OX-2 MOF, in which the photoexcitation at 315$340 \mathrm{~nm}$ induces an intense emission with a well-resolved vibrational structure having maxima at 480,520 and $560 \mathrm{~nm} .{ }^{40}$ This emission was previously assigned to a ligand-to-metal charge transfer (LMCT) phenomenon. ${ }^{40}$ However, the luminescent quantum yield of pristine OX-2 was higher (45\%) than that of the transformed Ag/OX-1 material, suggesting that the OX-1 large crystals are absorbing part of the irradiating light and therefore, diminishing the quantum yield (as they are not luminescent). Interestingly, we found that the emission of these new materials is very sensitive to the presence of acetone. While the emission of $\mathrm{Ag} / \mathrm{OX}-1$ exhibits smaller changes in many different solvents, its emission almost disappears in the presence of acetone ( $\sim 90 \%$ quenched emission), as shown in Fig. 4B and 4D. These are very relevant results as the detection of acetone in human breath is a highly promising alternative to commercially available methods for the diagnosis and monitoring of type 1 diabetes mellitus (such as urine or blood tests), which are more invasive, painful and could be unsafe. ${ }^{33}$ It is well-known that when the level of insulin is insufficient, the human body cannot convert the glucose into energy and instead, the cells burn fats, generating acetone during the process. ${ }^{32,33}$ The levels of acetone for a healthy individual ranges from 0.2-1.8 ppm while patients suffering a diabetes episode can exhale from 1.25 to 25 ppm of acetone. ${ }^{31,32}$ Therefore, the ability to detect small amounts of acetone is paramount, stimulating the development of efficient sensors to enable the accurate detection and precise management of diabetes mellitus. Diverse methods have been proposed, however most of them are expensive, non-portable, or require operation in high temperatures (e.g. oxide-based sensors). ${ }^{33,44,45}$ An alternative attracting much attention is based on fluorometric sensors, ${ }^{30,46-48}$ which can be of lower cost, portable, highly sensitive, and present a faster response. ${ }^{3}$ Although 
more tests must be done (e.g., vapour sensing studies), the selective response of Ag/OX-1 materials towards acetone positions them as a promising candidate for deployment as a fluorometric acetone sensor.

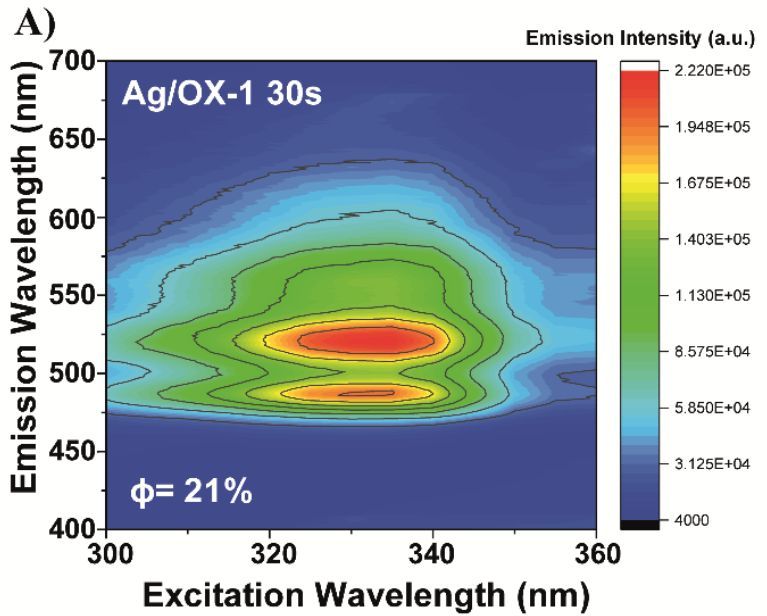

C)

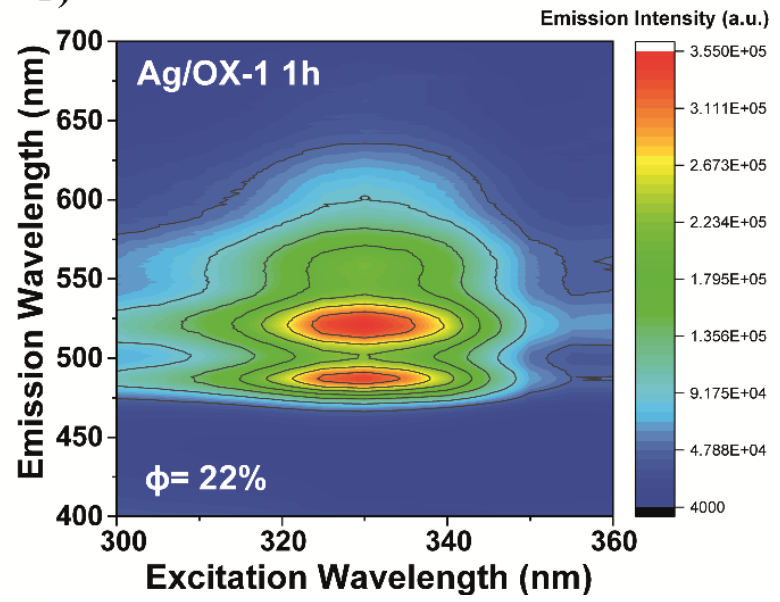

B)

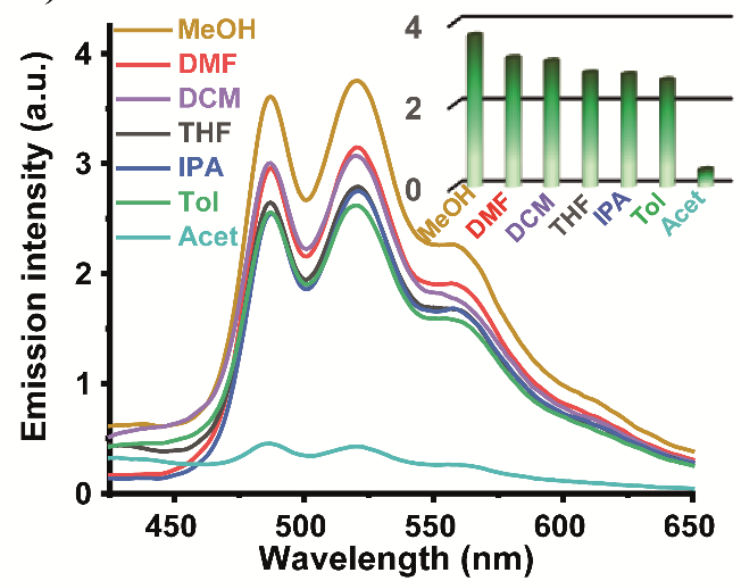

D)

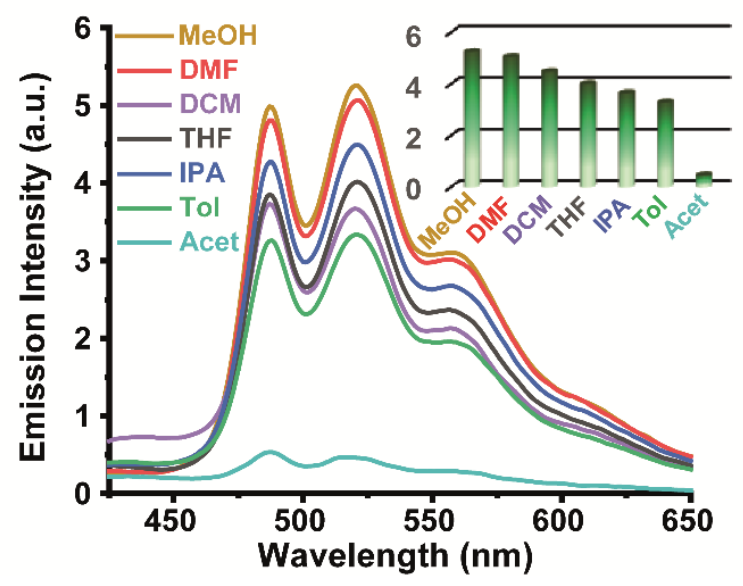

Figure 4. Excitation-emission maps of A) Ag/OX-1 $30 \mathrm{~s}$ and C) $\mathrm{Ag} / \mathrm{OX}-11 \mathrm{hr}$. The inset shows the emission quantum yield of each sample upon excitation at $330 \mathrm{~nm}$. Emission spectra of B) Ag/OX-1 $30 \mathrm{~s}$ and D) Ag/OX-1 $1 \mathrm{hr}$ in the presence of different VOCs. The inset is a graphical representation of the emission intensity maximum of Ag/OX-1 materials in each solvent, reflecting the significant quenching detected in acetone. The samples were excited at $330 \mathrm{~nm}$. 


\section{One-pot synthesis of $\mathrm{OX}-1$ and $\mathrm{OX}-2$ mixture}

To investigate whether the materials derived from the transformation of $\mathrm{OX}-1$ to $\mathrm{OX}-2$ is distinct from a direct production, we have synthesized a mixture of OX-1 and OX-2 in a one-pot reaction (see experimental part). SEM micrographs and the corresponding EDX maps reveal the co-existence of both OX-1 and OX-2 crystals (Fig. S6A-E). Similar to the Ag/OX-1 materials, in the one-pot synthesis, the OX-1 crystals are relatively larger than the OX-2 crystals. In addition, however, important structural changes were observed. Contrary to the transformed Ag/OX-1 materials, where the Bragg peak of OX-1 at $2 \theta$ angle of $5^{\circ}$ vanishes (suggesting the loss of some long-range periodicity), this peak contributes strongly to the signal in the mixture obtained through a one-pot synthesis (Fig. S6F). Moreover, all the diffraction peaks corresponding to OX-1 and OX-2 crystalline structures are identified in the PXRD pattern (Fig. S6F). This superposition suggests the coexistence of two-well differentiated phases of OX-1 and OX-2 crystals.

Fig. S6G displays the emission map of the material mixture, where, upon excitation at 315-340 $\mathrm{nm}$, a well-resolved vibrational emission band with maxima at 480, 520 and $560 \mathrm{~nm}$ is observed. Interestingly, the quantum yield is now $45 \%$ which is exactly the same value as for the pristine OX-2 MOF synthesized in methanol. ${ }^{40}$ This supports the fact that in the one-pot reaction, two distinguishable phases of OX-1 and OX-2 MOFs were obtained, as opposed to the one obtained through the transformation. This also affects the ability to detect acetone. While the transformed $\mathrm{Ag} / \mathrm{OX}-1$ materials are very sensitive to the presence of acetone, the mixture does not achieve the same sensing capabilities (Fig. S6H-I). Although the emission of the related mixture is quenched in the presence of acetone, it is just reduced by $60 \%$ (compared to the highest value), very far from the $90 \%$ of quenching observed for the $\mathrm{Ag} / \mathrm{OX}-1$ transformed materials. Additionally, the emission quenching of the mixture in the presence of acetone is not very different from the one observed 
for toluene or DCM (Fig. S6H-I), indicating that this mixture is much less selective towards acetone.

\section{Environmentally Friendly Transformations of OX-1 to OX-2}

With a view of fabricating the transformed $\mathrm{Ag} / \mathrm{OX}-1$ materials through an eco-friendlier route, we have explored new alternatives like a mechanochemical transformation or the use of deionized water instead of methanol. The transformation of OX-1 to OX-2 in deionized water was performed by mixing powders of $\mathrm{OX}-1(250 \mathrm{mg})$ and $\mathrm{AgNO}_{3}(120 \mathrm{mg}, 0.7 \mathrm{mmol})$ followed by the addition of small amounts of deionized water $(50-250 \mu \mathrm{L})$ and a brief mixing of all the reactants (see experimental part). As OX-1 is not very stable in water, we applied the same methodology to pristine OX-1 (addition of $250 \mu \mathrm{L}$ of deionized water to $250 \mathrm{mg}$ of OX-1) to test whether OX-1 degrades under these conditions. As shown in Fig. S7A, the PXRD spectrum of OX-1 after the addition of $250 \mu \mathrm{L}$ water maintains the main characteristic Bragg diffraction peaks, indicating that no important structural changes occur upon the addition of such small amounts of water. On the other hand, in the presence of $\mathrm{AgNO}_{3}$ and water, the OX-1 is transformed to OX-2 in a similar way to that described in methanol. The PXRD spectra show a combination of OX-1 and OX-2 diffraction peaks, but the OX-1 peak at $2 \theta$ angle of $5^{\circ}$ disappears (Fig. S7A). The FTIR spectra (Fig. S7B) yield very similar results to those described above, where the bands in the lower energy region remain unaffected, while the bands at $\sim 1600$ and $\sim 1390 \mathrm{~cm}^{-1}$ are strongly shifted to lower energies, agreeing with a coordination between BDC and Ag. Similar results were observed for the materials obtained through the mechanochemical OX-1 to OX-2 transformation. In this case, $250 \mathrm{mg}$ of $\mathrm{OX}-1$ and $120 \mathrm{mg}$ of $\mathrm{AgNO}_{3}$ were ground by mortar and pestle. For one batch, the 
powders were ground for $30 \mathrm{~min}$, while in a second batch, another mixture was ground for $2 \mathrm{~min}$ after the addition of one drop of water in order to facilitate the reaction (see experimental part). In both cases, the PXRD spectra show the combination of OX-1 and OX-2 diffraction peaks, along with the disappearance of the OX-1 peak at $2 \theta$ angle of $5^{\circ}$ (Fig. S8A), and the FTIR spectra illustrate the shift of the bands at $\sim 1600$ and $\sim 1390 \mathrm{~cm}^{-1}$ to lower energies (Fig. S8B).

As expected, the transformed Ag/OX-1 materials exhibit a green luminescent emission. As an example, Fig. S8C-D displays a photo of the transformed materials upon UV (365 nm) irradiation. First, the OX-1 MOF is not emissive in the visible spectral range, but during the grinding process, the mixture becomes luminescent due to the rapid transformation of OX-1 to OX-2 (Fig. S8C). In a similar way, the addition of 1 drop of water produces an instantaneous reaction, which facilitates the mechanochemical transformation process (Fig. S8D). The excitation-emission maps of the $\mathrm{Ag} / \mathrm{OX}-1$ transformed materials in water are comparable to the ones of the materials synthesized in methanol (Fig. S9). Interestingly, the fluorescence quantum yield value increases with the amount of water used (from 12 to $16 \%$ ), presumably because more OX-1 is being transformed into OX-2. The transformed $\mathrm{Ag} / \mathrm{OX}-1\left(250 \mu \mathrm{L} \mathrm{H}_{2} \mathrm{O}\right)$ material exhibits an astonishing response to acetone. In the presence of this volatile organic compound (VOC), the emission intensity is quenched by almost 100\%, while it is barely affected by the presence of other VOCs (Fig. S9D). The materials obtained through the mechanochemical procedure unveil excitation-emission maps (Fig. S10A-B) that are comparable to those of Fig. 4A,C. However, the emission quantum yield is relatively lower (3-5\%), indicating that the mechanochemical transformation is less effective. The excellent ability of this $\mathrm{Ag} / \mathrm{OX}-1$ material to selectively detect acetone is presented in Fig. S10C-D. Based on the above results, we not only demonstrate the possibility of transforming OX- 
1 to OX-2 by employing a green methodology, but more importantly, we unravel that the obtained materials could become promising candidates for developing acetone sensors.

\section{Transformation of $\mathrm{OX}-1$ to $\mathrm{Pb}-\mathrm{MOF}$}

To exploit and further demonstrate the versatility of the MOF transformation mechanism described above, we explored the conversion of OX-1 to other type of MOF incorporating a different metal ion. We selected $\mathrm{Pb}$ as the secondary metal ion based on two main reasons: (i) firstly because it has been previously reported that Pb-BDC MOFs are luminescent, ${ }^{49}$ and thus, the transformation could be easily followed by irradiating the mixture with a UV light, and (ii) secondly and most importantly, $\mathrm{Pb}$ metal ions are a highly toxic pollutant that can be found in aquatic environments. ${ }^{34,35}$ Therefore, if OX-1 can be quickly transformed to a Pb-MOF, it could be an interesting approach to capture $\mathrm{Pb}$ ions from polluted water.

We followed the same methodology described above for the Ag/OX-1 materials, but now 250 $\mathrm{mg}$ of $\mathrm{OX} 1$ were soaked in a solution of $165 \mathrm{mg}(0.5 \mathrm{mmol})$ of $\mathrm{Pb}\left(\mathrm{NO}_{3}\right)_{2}$ in $20 \mathrm{~mL}$ of $\mathrm{MeOH}$ for 20 min (see experimental part). Fig. 5A shows the PXRD patterns of pristine OX-1 and Pb-BDC MOFs and the $\mathrm{Pb} / \mathrm{OX}-1$ transformed material. Remarkably, the PXRD spectrum of the transformed $\mathrm{Pb} / \mathrm{OX}-1$ matches that of the pristine $\mathrm{Pb}-\mathrm{BDC} \mathrm{MOF},{ }^{50-52}$ and in contrary to the Ag/OX-1 materials, no features of OX-1 diffraction Bragg peaks were observed. This fact implies a complete transformation of $\mathrm{OX}-1$ to the $\mathrm{Pb}-\mathrm{BDC}$ MOF in the first 20 min, suggesting a higher affinity towards $\mathrm{Pb}$ ions than to the $\mathrm{Ag}$ ones. As expected, this transformation produces a luminescent $\mathrm{Pb}-\mathrm{BDC}$ MOF (quantum yield of $8 \%$ ), whose emission is characterized by a broad band with an intensity maximum at around $500 \mathrm{~nm}$ upon excitation at 315-350 nm (Fig. 5B). 

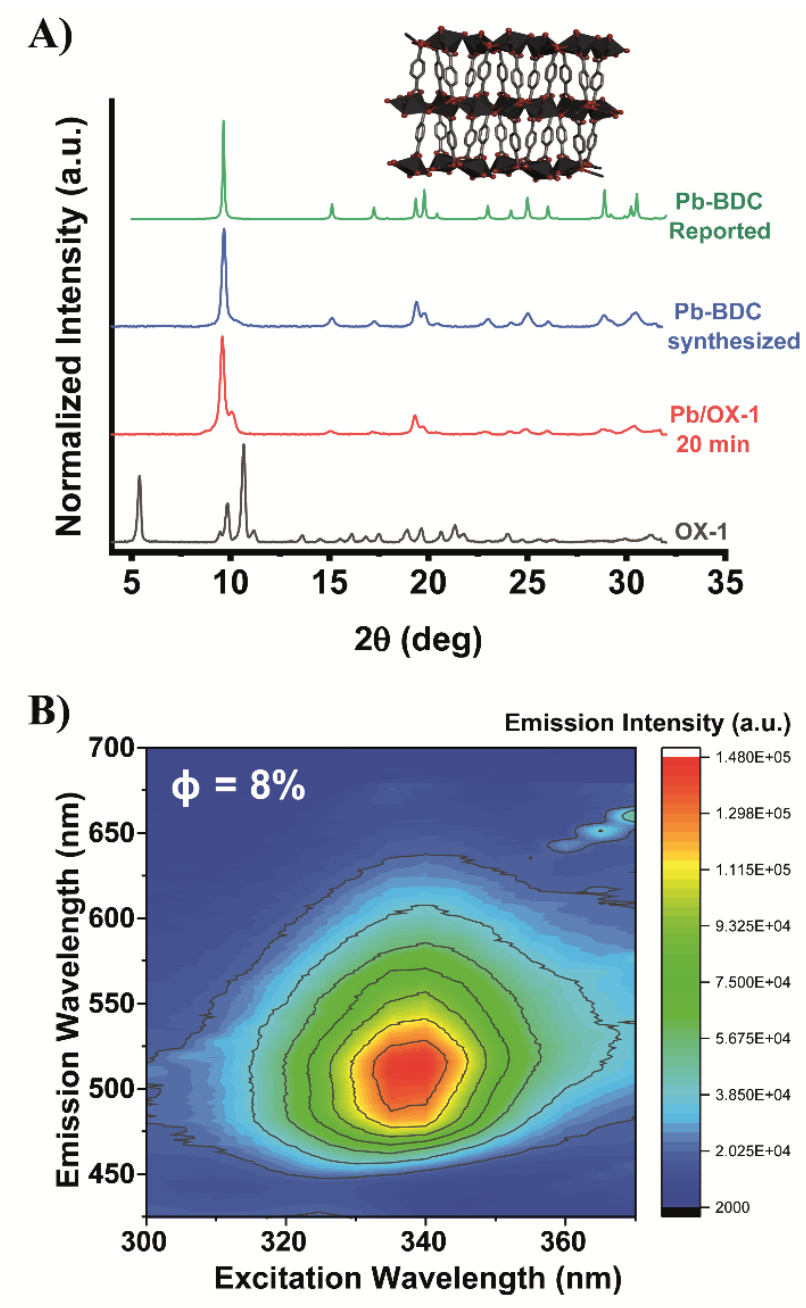

Figure 5. A) PXRD patterns of pristine $\mathrm{OX}-1$ and $\mathrm{Pb}-\mathrm{BDC}$ (as-synthesized and reported) MOFs and the converted $\mathrm{Pb} / \mathrm{OX}-1$ material. B) Excitation-emission map of $\mathrm{Pb} / \mathrm{OX}-1$ material. The inset is the emission quantum yield of the sample upon excitation at $330 \mathrm{~nm}$.

The extremely fast and complete transformation of $\mathrm{OX}-1$ to $\mathrm{Pb}-\mathrm{BDC}$ MOF encourages us to exploit this mechanism for the capturing of $\mathrm{Pb}$ ions in water. However, some important aspects must be considered prior to that. Firstly, the synthesis of Pb-BDC MOF in water generates a different MOF structure, ${ }^{53}$ and therefore the OX-1 conversion could be different. Secondly and more crucially, OX-1 MOF degrades when immersed in water, hindering its possible applicability for $\mathrm{Pb}$ trapping from aquatic media. To overcome the latter issue, we have dispersed the OX-1 MOF in a hydrophobic polyurethane membrane (OX-1/PU see experimental part). The fabrication of mixed-matrix membranes (MMM) composed of a MOF and a polymer is a well-established 
procedure for boosting the use of MOFs in disruptive technologies in applications such as gas or liquid separation or water purification. ${ }^{54-58}$ The idea behind this is to synergistically combine the excellent properties of MOFs (high surface area, long range periodicity, etc.) and polymers (ease of processing and tunable mechanical properties). ${ }^{59-61}$ In our case, we envisaged that the polyurethane membrane (due to its rubbery nature) ${ }^{62}$ will permit a controlled diffusion of the $\mathrm{Pb}$ water solution, thus preventing the degradation of OX-1 MOF. This assumption was further corroborated by PXRD experiments (Fig. S11), which showed that the crystalline structure of OX1 in PU remains stable after its immersion in water for 20 mins (duration of the experiment). In contrast, we note that the pristine OX-1 instantaneously degrades in water (when not protected within PU matrix).

Once we established that OX-1/PU MMM is resilient enough to water exposure, we investigated whether the OX-1 in the composite membrane can be transformed to Pb-BDC MOF under these conditions, and therefore, whether the $\mathrm{OX}-1 / \mathrm{PU}$ membrane is capable of capturing $\mathrm{Pb}$ ions from a water solution. To proof this concept, a film of OX-1/PU (size: $\sim 2.5 \times 2.5 \mathrm{~cm}^{2}$ ) was immersed in a solution of $\mathrm{Pb}\left(\mathrm{NO}_{3}\right)_{2}$ in water $(0.05 \mathrm{M})$ without stirring for 20 mins. The film was then dried at room temperature; and the conversion of $\mathrm{OX}-1$ to $\mathrm{Pb}-\mathrm{BDC}$ MOF was confirmed by PXRD and luminescence experiments. Fig. 6A shows the PXRD spectra of OX-1/PU, pristine Pb-BDC- $\mathrm{H}_{2} \mathrm{O}$ (synthesized in water) and the transformed Pb-OX-1/PU. The PXRD pattern of the latter exhibits the Bragg diffraction peaks characteristic of $\mathrm{Pb}-\mathrm{BDC}-\mathrm{H}_{2} \mathrm{O},{ }^{53}$ indicating a conversion from $\mathrm{OX}-1$ to $\mathrm{Pb}-\mathrm{BDC} \mathrm{MOF}$. The $\mathrm{OX}-1$ to $\mathrm{Pb}-\mathrm{BDC}$ conversion was also confirmed by the turquoise luminescence of the Pb-OX-1/PU membrane. Fig. 6B displays the photos of the as-synthesized $\mathrm{OX}-1 / \mathrm{PU}$ film prior to and after its interaction with $\mathrm{Pb}$ ions under $\mathrm{UV}(365 \mathrm{~nm})$ lamp irradiation. The pristine OX-1/PU membrane exhibits a light blue emission typical of the PU membrane. On 
the contrary, the $\mathrm{Pb}-\mathrm{OX}-1 / \mathrm{PU}$ membrane unveils a turquoise emission, whose intensity is gradually increasing with the time exposed to the $\mathrm{Pb}$ solution. Fig. $6 \mathrm{C}$ shows the emission spectra of the pristine $\mathrm{OX}-1 / \mathrm{PU}$ and the converted $\mathrm{Pb}-\mathrm{OX}-1 / \mathrm{PU}$ membranes. The emission spectrum of the former, attributed to the PU polymer, consists of a broad band with maximum located at
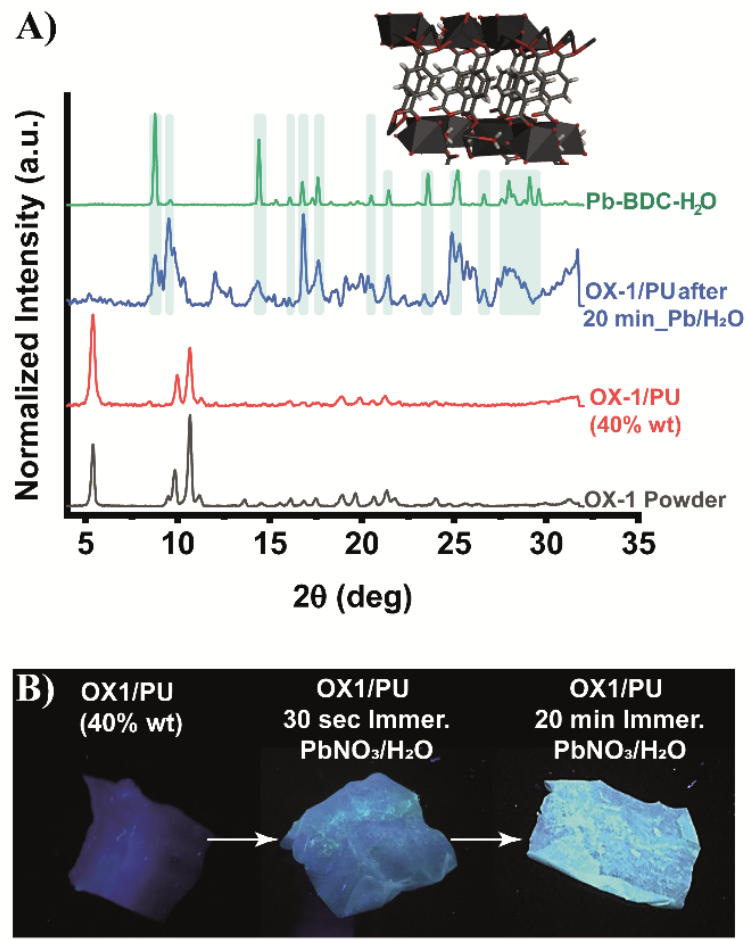

C)

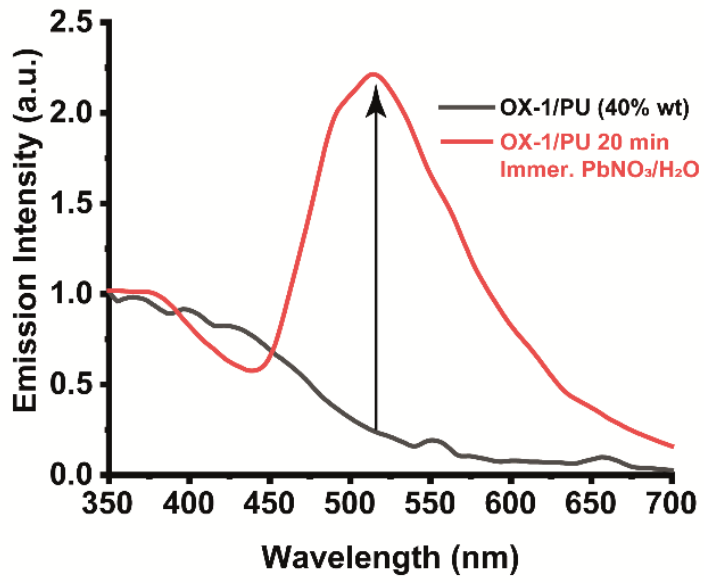

Figure 6. A) PXRD patterns of pristine $\mathrm{OX}-1$ and $\mathrm{Pb}-\mathrm{BDC}-\mathrm{H}_{2} \mathrm{O}$ MOF powders, and the OX-1/PU mixedmatrix membrane before and after immersing in a solution of $\mathrm{Pb}\left(\mathrm{NO}_{3}\right)_{2}$ for 20 minutes. B) Photo of the OX1/PU membrane illuminated with an UV lamp $(365 \mathrm{~nm})$ before and after its exposure to a solution of $\mathrm{Pb}\left(\mathrm{NO}_{3}\right)_{2}$ for 30 seconds and 20 minutes, respectively. C) Emission spectra of the OX-1/PU membrane before and after its submersion in a solution of $\mathrm{Pb}\left(\mathrm{NO}_{3}\right)_{2}$ for 20 minutes. The samples were excited at $330 \mathrm{~nm}$. 
$\sim 375 \mathrm{~nm}$. The emission spectrum of the Pb-OX-1/PU membrane depicts a similar emission band (at $375 \mathrm{~nm}$ ) of the PU along with a main band at $\sim 515 \mathrm{~nm}$, corresponding to the emission of the converted $\mathrm{Pb}-\mathrm{BDC}-\mathrm{H}_{2} \mathrm{O}$ MOF. Albeit preliminary, these results open an exciting pathway for the use of $\mathrm{OX}-1$ mixed-matrix membranes to capture $\mathrm{Pb}$ ions from aquatic environments. Moreover, the fact that OX-1 can be converted to different MOFs using such a different cation such as Ag or $\mathrm{Pb}$, leads us to believe that this methodology could be expanded to other metal cations or pollutants which can strongly interact with the uncoordinated oxygen atoms in the OX-1 MOF.

\section{Experimental Section}

\section{Materials}

Terephthalic acid $(\mathrm{BDC}, 99 \%+)$, zinc nitrate hexahydrate $\left(\mathrm{Zn}\left(\mathrm{NO}_{3}\right)_{2} \cdot 6 \mathrm{H}_{2} \mathrm{O}\right)$, zinc acetate dihydrate $\left(\mathrm{Zn}(\mathrm{OAc})_{2} \cdot 2 \mathrm{H}_{2} \mathrm{O}\right)$, silver nitrate $\left(\mathrm{AgNO}_{3}\right)$, lead nitrate $\left(\mathrm{Pb}\left(\mathrm{NO}_{3}\right)_{2}\right)$, triethylamine $\left(\mathrm{NEt}_{3}\right.$, 99\%), N, N-dimethylformamide (DMF, 99\%), dichloromethane (DCM, 99\%+), acetone (Acet, 99.6\% for spectroscopy), tetrahydrofuran (THF, 99.85\%), isopropanol (IPA, 99.5\%+), toluene $(\mathrm{Tol}, 99 \%+)$, and methanol $(\mathrm{MeOH}, 99.8 \%+)$ were purchased from Fisher Scientific and used without further purification.

\section{Synthesis of OX-1 MOF}

The OX-1 MOF was synthesized using the protocol described elsewhere with some modifications. ${ }^{30} 12.0 \mathrm{mmol}$ of BDC were deprotonated in a solution of $24.0 \mathrm{mmol}^{\circ} \mathrm{NEt}_{3}$ in 20 $\mathrm{mL}$ of methanol. A second solution was prepared by sonicating $6.0 \mathrm{mmol}$ of $\mathrm{Zn}\left(\mathrm{NO}_{3}\right)_{2} \cdot 6 \mathrm{H}_{2} \mathrm{O}$ in $20 \mathrm{~mL}$ of methanol. The latter solution was quickly added to the former and a white suspension was promptly formed. The mixture was sonicated for 5 minutes and then the white solid sample 
was washed twice with methanol, collected by centrifugation $(8000 \mathrm{rpm})$ and dried at $80{ }^{\circ} \mathrm{C}$ for 4 hours. This procedure yielded $2.5 \mathrm{~g}$ of OX-1 MOF.

\section{Conversion of OX-1 to OX-2 in methanol}

$0.7 \mathrm{mmol}$ of $\mathrm{AgNO}_{3}$ were sonicated until the complete dissolution of the crystals in $20 \mathrm{~mL}$ of methanol. Then, $250 \mathrm{mg}$ of OX-1 were poured into the above solution and the mixture was stirred for a certain time. Four different samples were prepared, stopping the reaction at $30 \mathrm{sec}, 5 \mathrm{mins}, 1$ $\mathrm{hr}$ and $24 \mathrm{hr}$, respectively. Then the solvent was removed, and the sample was briefly washed once with methanol to remove the possible excess of $\mathrm{Ag}$ ions. The sample was finally dried at $80{ }^{\circ} \mathrm{C}$ for 4 hours.

\section{Synthesis of MOF-5}

MOF-5 was synthesized following the recipe previously reported with some minor modifications. ${ }^{42} 15 \mathrm{mmol}$ of BDC were dissolved in a solution of $30 \mathrm{mmol}_{\text {of }} \mathrm{NEt}_{3}$ in $200 \mathrm{~mL}$ of DMF. Another solution was prepared by dissolving $38 \mathrm{mmol}$ of $\mathrm{Zn}(\mathrm{OAc})_{2} \cdot 2 \mathrm{H}_{2} \mathrm{O}$ in $200 \mathrm{~mL}$ of DMF. The zinc salt solution was added to the BDC one and the mixture was stirred for $2.5 \mathrm{hr}$. The white solid sample was washed twice with DMF and DCM, collected by centrifugation ( $8000 \mathrm{rpm})$ and dried at $120^{\circ} \mathrm{C}$ under reduced pressure for 4 hours. This procedure yielded $\sim 2.5 \mathrm{~g}$ of MOF-5.

\section{One-pot synthesis of OX-1/OX-2 mixture}

For the one-pot synthesis of the OX-1/OX-2 mixture, we followed a very similar methodology as for the synthesis of OX-1. Briefly, $6.0 \mathrm{mmol}$ of BDC were deprotonated in a solution of $12.0 \mathrm{mmol}$ of $\mathrm{NEt}_{3}$ in $20 \mathrm{~mL}$ of methanol. Another solution was prepared by sonicating $1.5 \mathrm{mmol}$ of $\mathrm{Zn}\left(\mathrm{NO}_{3}\right)_{2} \cdot 6 \mathrm{H}_{2} \mathrm{O}$ and $1.5 \mathrm{mmol}$ of $\mathrm{AgNO}_{3}$ in $20 \mathrm{~mL}$ of methanol. The second solution containing the mixture of metal ions was quickly added to the BDC one and a white suspension was promptly 
formed. The mixture was sonicated for 10 mins and the white solid sample was washed twice with methanol, collected by centrifugation $(8000 \mathrm{rpm})$ and dried at $80{ }^{\circ} \mathrm{C}$ for $4 \mathrm{hr}$.

\section{Green conversion of OX-1 to OX-2: Mechanochemistry and deionized water}

Mechanochemistry: $250 \mathrm{mg}$ of OX-1 and $0.7 \mathrm{mmol}$ of $\mathrm{AgNO}_{3}$ were ground by mortar and pestle for 30 mins (the humidity of the lab was $40 \%$ ). The conversion was followed by irradiating the sample with UV light (Fig. S8C). The white solid sample was then dried at $100{ }^{\circ} \mathrm{C}$ for $2 \mathrm{~h}$. A similar procedure was followed but with the addition of 1 drop of water in order to accelerate the grinding process. In this case the mixture was only ground for two minutes and a similar degree of OX-1 to OX-2 conversion was observed. As in the above example, the sample was dried at $100{ }^{\circ} \mathrm{C}$ for $2 \mathrm{~h}$.

Water methodology: 3 different samples were prepared by the addition of increasing amounts of deionized water $(50,100$ and $250 \mu \mathrm{L})$ to a mixture of $250 \mathrm{mg}$ of OX-1 and $0.7 \mathrm{mmol}$ of $\mathrm{AgNO}_{3}$. The reactants were mixed for 1 min using a spatula, and the white paste was dried at $100{ }^{\circ} \mathrm{C}$ for 2 hr.

\section{Conversion of OX-1 to Pb-BDC MOF in methanol}

This procedure was similar to the one described above. Briefly, $0.5 \mathrm{mmol}$ of $\mathrm{Pb}\left(\mathrm{NO}_{3}\right)_{2}$ were sonicated in $20 \mathrm{ml}$ of methanol. Then, $250 \mathrm{mg}$ of OX-1 were poured in the former solution and the mixture was stirred for 20 minutes. After that, the solvent was removed, and the sample was washed once with methanol to remove any excess $\mathrm{Pb}$ ions. The sample was finally dried at $80{ }^{\circ} \mathrm{C}$ for $4 \mathrm{hr}$. 


\section{Fabrication of the OX-1/PU mixed-matrix membrane (MMM)}

The OX-1/PU MMM was fabricated similarly to previous reports. ${ }^{63,64}$ Firstly, a PU solution $(15 \% \mathrm{w} / \mathrm{w})$ was prepared by dissolving poly [4,4'-methylenebis(phenyl isocyanate)-alt-1,4butanediol/di(propylene glycol)/polyurethane] pellets (Sigma Aldrich) in THF by stirring the mixture for $48 \mathrm{hr}$ until the complete dissolution of the polymer pellets. Subsequently, a certain amount of OX-1 was dispersed in a small amount of THF $(1 \mathrm{~mL})$ by sonicating and stirring the suspension. This OX-1 dispersion was then incorporated in the PU-THF solution to yield 40 wt.\% of MOF. The wt.\% of MOF in the PU matrix was determined using the following equation:

$$
\text { MOF wt. } \%=\left(\frac{m_{\mathrm{MOF}}}{m_{\mathrm{MOF}}+m_{\mathrm{PU}}}\right) \times 100
$$

where $m_{\mathrm{MOF}}$ and $m_{\mathrm{PU}}$ are the weights of OX-1 MOF (dispersed in THF) and PU (dissolved in THF), respectively.

The MMM was finally obtained by doctor blading the OX-1/PU mixture (in THF), following by the drying of the composite film at room temperature. The membrane has an original size of around $10 \times 10 \mathrm{~cm}^{2}$ and a thickness of $\sim 0.5 \mathrm{~mm}$. For the $\mathrm{Pb}$ capture experiments the membrane was cut to a size of $\sim 2.5 \times 2.5 \mathrm{~cm}^{2}$.

\section{Materials characterization}

The crystalline structure, morphology and luminescent properties of the materials were characterized by a combination of powder X-ray (PXRD), Fourier transformed infrared (FTIR) and fluorescence spectroscopy and field emission scanning electron microscopy coupled to energy dispersive X-ray (FESEM-EDX) spectroscopy techniques. PXRD experiments were conducted in a Rigaku Miniflex diffractometer with a $\mathrm{Cu} \mathrm{K \alpha}$ source $(1.541 \AA)$. The diffraction data were collected using $0.01^{\circ}$ step size, $1^{\circ} \mathrm{min}^{-1}$ and for $2 \theta$ angle ranging from $2^{\circ}$ to $32^{\circ}$. FESEM images 
and the corresponding EDX spectra were obtained using the Carl Zeiss Merlin equipped with a field emission gun. Micrographs were attained under high vacuum with an accelerating voltage of $10 \mathrm{keV}$ and in secondary electron imaging mode. FTIR spectra were recorded on a Nicolet iS10 spectrometer. The FTIR spectrum of each sample was collected 3 times and then averaged.

Near-field spectroscopy and AFM imaging were performed on a neaSNOM instrument (Neaspec $\mathrm{GmbH}$ ) based on a tapping-mode AFM. Here, the platinum-coated tip (oscillation frequency $\Omega=254 \mathrm{kHz}$ ) is used both to map the height topography and to probe the optical nearfield upon illumination with a broadband mid-infrared laser source (Toptica, Germany). The detector signal is demodulated at higher harmonics $(n \Omega)$ to suppress the interference of background contributions. Each spectrum is acquired by Fourier transform spectroscopy averaging over 10 individual interferograms with an integration time of $14 \mathrm{~ms}$, probed with a spot size of 20 $\mathrm{nm}$ on individual crystals. All spectra are normalized by reference spectra obtained on the silicon substrate.

Steady-state fluorescence spectra, excitation-emission maps, and luminescence quantum yields were recorded using a FS5 spectrofluorometer (Edinburgh Instruments) equipped with different modules for each specific experiment (i.e. integrating sphere for quantum yield, or modules for liquid or solid-samples).

For the emission experiments of $\mathrm{Ag} / \mathrm{OX}-1$ in presence of different VOCs, $2 \mathrm{mg}$ of the MOF material was added to $4 \mathrm{~mL}$ of each solvent. The mixture was sonicated for $15 \mathrm{~min}$ to disperse well the MOF crystals. All the experiments were performed at room temperature. 


\section{Conclusions}

In this study, we have established a post-synthetic approach to accomplish MOF-toMOF transformation by leveraging the uncoordinated oxygen positions present in the BDC linkers of the OX-1 MOF. The conversion reaction is facile and fast, requiring only the immersion of a certain amount of OX-1 MOF in a silver metal salt solution for a short period of time ( $\sim$ minutes $)$. We also demonstrated that the MOF transformation can be extended to green methodologies involving dry media through a mechanochemical reaction (grinding mechanism), or simply through the use of water (instead of methanol). The obtained Ag/OX-1 materials exhibit an intense green emission, which is selectively quenched in the presence of acetone, making them promising candidates for the future development of advanced sensor devices for diabetes monitoring. The underlying mechanism for acetone sensing is currently unknown and it warrants further investigation. Our protocol was also exploited for the rapid fully conversion of $\mathrm{OX}-1$ to different $\mathrm{Pb}-\mathrm{BDC}$ MOFs. This finding motivated us to prepare a mixed-matrix membrane by dispersing the OX-1 in a rubbery polyurethane membrane which was then employed as a proof-of-concept for capturing $\mathrm{Pb}$ ions from a water solution. Our work highlights the use of alternative synthetic routes to impart desired qualities to MOFs like luminescence, or even to take advantage of these approaches for emerging applications such as acetone sensing or water purification technologies. 


\section{AUTHOR INFORMATION}

\section{Corresponding Author}

*Jin-Chong Tan.

E-mail: jin-chong.tan@eng.ox.ac.uk

\section{Funding Sources}

This work was supported by the ERC Consolidator Grant through the grant agreement 771575 (PROMOFS).

\section{Supporting Information}

Supporting Information includes (i) FESEM-EDX images of the OX-1, Ag/OX-1, and OX-1/OX2 materials; (ii) the excitation-emission maps of $\mathrm{Ag} / \mathrm{OX}-1$ at 5min and 24h; (iii) PXRD patterns, excitation-emission maps, and luminescence response to different VOCs of the OX-1/OX-2 mixture; (iv) PXRD and FTIR spectra of Ag/OX-1 materials transformed using water; (v) PXRD and FTIR spectra together with photographic images of the mechanochemically transformed Ag/OX-1 materials; (vi) excitation-emission maps, and luminescence response to different VOCs of Ag/OX-1 (transformed mechanochemically) and using water, respectively; and (vii) PXRD patterns of OX-1/PU membranes before and after water immersion study.

\section{Acknowledgements}

We thank the Research Complex at Harwell (RCaH) for access to the Nicolet iS10 FTIR spectrometer. We thank Dr. Barbara Souza and Dr. Jennifer Holter for their help with FESEMEDX characterization. 


\section{REFERENCES}

1. Zhou, H.-C.; Long, J. R.; Yaghi, O. M. Introduction to Metal-Organic Frameworks. Chem. Rev. 2012, $112(2), 673-674$.

2. $\quad$ Burtch, N. C.; Heinen, J.; Bennett, T. D.; Dubbeldam, D.; Allendorf, M. D. Mechanical Properties in Metal-Organic Frameworks: Emerging Opportunities and Challenges for Device Functionality and Technological Applications. Adv. Mater. 2018, 30 (37), article no. 1704124.

3. Lustig, W. P.; Mukherjee, S.; Rudd, N. D.; Desai, A. V.; Li, J.; Ghosh, S. K. MetalOrganic Frameworks: Functional Luminescent and Photonic Materials for Sensing Applications. Chem. Soc. Rev. 2017, 46 (11), 3242-3285.

4. Zhang, Z.; Sang, W.; Xie, L.; Dai, Y. Metal-Organic Frameworks for Multimodal Bioimaging and Synergistic Cancer Chemotherapy. Coord. Chem. Rev. 2019, 399, article no. 213022.

5. Stock, N.; Biswas, S. Synthesis of Metal-Organic Frameworks (MOFs): Routes to Various MOF Topologies, Morphologies, and Composites. Chem. Rev. 2012, 112 (2), 933-969.

6. Tanabe, K. K.; Cohen, S. M. Postsynthetic Modification of Metal-Organic Frameworksa Progress Report. Chem. Soc. Rev. 2011, 40 (2), 498-519.

7. Wang, Z.; Cohen, S. M. Postsynthetic Modification of Metal-Organic Frameworks. Chem. Soc. Rev. 2009, 38 (5), 1315-1329.

8. Yin, Z.; Wan, S.; Yang, J.; Kurmoo, M.; Zeng, M.-H. Recent Advances in Post-Synthetic Modification of Metal-Organic Frameworks: New Types and Tandem Reactions. Coord. Chem. Rev. 2019, 378, 500-512.

9. Burrows, A. D., Metal-Organic Frameworks: Postsynthetic Modification. In Encyclopedia of Inorganic and Bioinorganic Chemistry, John Wiley \& Sons, Ltd.: 2014; pp 1-24.

10. Kandiah, M.; Usseglio, S.; Svelle, S.; Olsbye, U.; Lillerud, K. P.; Tilset, M. PostSynthetic Modification of the Metal-Organic Framework Compound UiO-66. J. Mater. Chem. 2010, 20 (44), 9848-9851.

11. Kim, M.; Cahill, J. F.; Prather, K. A.; Cohen, S. M. Postsynthetic Modification at Orthogonal Reactive Sites on Mixed, Bifunctional Metal-Organic Frameworks. Chem. Commun. 2011, 47 (27), 7629-7631.

12. Lalonde, M.; Bury, W.; Karagiaridi, O.; Brown, Z.; Hupp, J. T.; Farha, O. K. Transmetalation: Routes to Metal Exchange within Metal-Organic Frameworks. J. Mater. Chem. A 2013, 1 (18), 5453-5468.

13. Brozek, C. K.; Dincă, M. Cation Exchange at the Secondary Building Units of MetalOrganic Frameworks. Chem. Soc. Rev. 2014, 43 (16), 5456-5467.

14. Mendes, R. F.; Almeida Paz, F. A. Transforming Metal-Organic Frameworks into Functional Materials. Inorg. Chem. Front. 2015, 2 (6), 495-509.

15. Abednatanzi, S.; Gohari Derakhshandeh, P.; Depauw, H.; Coudert, F.-X.; Vrielinck, H.; Van Der Voort, P.; Leus, K. Mixed-Metal Metal-Organic Frameworks. Chem. Soc. Rev. 2019, 48 (9), 2535-2565.

16. Aguirre-Díaz, L. M.; Gándara, F.; Iglesias, M.; Snejko, N.; Gutiérrez-Puebla, E.; Monge, M. Á. Tunable Catalytic Activity of Solid Solution Metal-Organic Frameworks in One-Pot Multicomponent Reactions. J. Am. Chem. Soc. 2015, 137 (19), 6132-6135.

17. Zhao, C.; Dai, X.; Yao, T.; Chen, W.; Wang, X.; Wang, J.; Yang, J.; Wei, S.; Wu, Y.; Li, Y. Ionic Exchange of Metal-Organic Frameworks to Access Single Nickel Sites for Efficient Electroreduction of CO2. J. Am. Chem. Soc. 2017, 139 (24), 8078-8081. 
18. Lee, Y.; Kim, S.; Kang, J. K.; Cohen, S. M. Photocatalytic CO2 Reduction by a Mixed Metal (Zr/Ti), Mixed Ligand Metal-Organic Framework Under Visible Light Irradiation. Chem. Commun. 2015, 51 (26), 5735-5738.

19. Navarro Amador, R.; Carboni, M.; Meyer, D. Sorption and Photodegradation Under Visible Light Irradiation of an Organic Pollutant by a Heterogeneous UiO-67-Ru-Ti MOF Obtained by Post-Synthetic Exchange. RSC Adv 2017, 7 (1), 195-200.

20. Kaur, G.; Rai, R. K.; Tyagi, D.; Yao, X.; Li, P.-Z.; Yang, X.-C.; Zhao, Y.; Xu, Q.; Singh, S. K. Room-Temperature Synthesis of Bimetallic Co-Zn Based Zeolitic Imidazolate Frameworks in Water for Enhanced CO2 and H2 Uptakes. J. Mater. Chem. A 2016, 4 (39), 1493214938.

21. Zhai, Q.-G.; Bu, X.; Mao, C.; Zhao, X.; Feng, P. Systematic and Dramatic Tuning on Gas Sorption Performance in Heterometallic Metal-Organic Frameworks. J. Am. Chem. Soc. 2016, 138 (8), 2524-2527.

22. Cui, Y.; Xu, H.; Yue, Y.; Guo, Z.; Yu, J.; Chen, Z.; Gao, J.; Yang, Y.; Qian, G.; Chen, B. A Luminescent Mixed-Lanthanide Metal-Organic Framework Thermometer. J. Am. Chem. Soc. 2012, 134 (9), 3979-3982.

23. Wang, Z.; Ananias, D.; Carné-Sánchez, A.; Brites, C. D. S.; Imaz, I.; Maspoch, D.; Rocha, J.; Carlos, L. D. Lanthanide-Organic Framework Nanothermometers Prepared by SprayDrying. Adv. Funct. Mater. 2015, 25 (19), 2824-2830.

24. Singha, D. K.; Bhattacharya, S.; Majee, P.; Mondal, S. K.; Kumar, M.; Mahata, P. Optical Detection of Submicromolar Levels of Nitro Explosives by a Submicron Sized Metal-Organic Phosphor Material. J. Mater. Chem. A 2014, 2 (48), 20908-20915.

25. Singha, D. K.; Majee, P.; Mondal, S. K.; Mahata, P. A Eu-Doped Y-Based Luminescent Metal-Organic Framework as a Highly Efficient Sensor for Nitroaromatic Explosives. Eur. J. Inorg. Chem. 2015, 2015 (8), 1390-1397.

26. Li, W.; Zhang, Y.; Zhang, C.; Meng, Q.; Xu, Z.; Su, P.; Li, Q.; Shen, C.; Fan, Z.; Qin, L.; Zhang, G. Transformation of Metal-Organic Frameworks for Molecular Sieving Membranes. Nat. Commun. 2016, 7 (1), article no. 11315.

27. Jonckheere, D.; Coutino, E.; Baekelant, W.; Bueken, B.; Reinsch, H.; Fenwick, O.; Richard, F.; Samori, P.; Ameloot, R.; Hofkens, J.; Roeffaers, M. B. J.; De Vos, D. E. SilverInduced Reconstruction of an Adeninate-Based Metal-Organic Framework for Encapsulation of Luminescent Adenine-Stabilized Silver Clusters. J. Mater. Chem. C 2016, 4, 4259-4268.

28. Burrows, A. D.; Cassar, K.; Friend, R. M. W.; Mahon, M. F.; Rigby, S. P.; Warren, J. E. Solvent Hydrolysis and Templating Effects in the Synthesis of Metal-Organic Frameworks. CrystEngComm 2005, 7 (89), 548-550.

29. Biemmi, E.; Bein, T.; Stock, N. Synthesis and Characterization of a New Metal Organic Framework Structure with a 2D Porous System: (H2NEt2)2[Zn3(BDC)4]·3DEF. Solid State Sci 2006, 8 (3), 363-370.

30. Chaudhari, A. K.; Kim, H. J.; Han, I.; Tan, J.-C. Optochemically Responsive 2D Nanosheets of a 3D Metal-Organic Framework material. Adv. Mater. 2017, 29 (27), article no. 1701463.

31. Amann, A. S., D. , Volatile Biomarkers. In Non-Invasive Diagnosis in Physiology and Medicine. Elsevier: New York, NY, USA,: 2013.

32. Rydosz, A. Sensors for Enhanced Detection of Acetone as a Potential Tool for Noninvasive Diabetes Monitoring. Sensors 2018, 18, article no. 2298. 
33. Saasa, V. M., T.; Beukes, M.; Mokgotho, M.; Liu, C.-P.; Mwakikunga, B. Sensing Technologies for Detection of Acetone in Human Breath for Diabetes Diagnosis and Monitoring. Diagnostics 2018, 8, 12.

34. Morgan, A. M., Shin, H. C., Abd El-Aty, A. M. Characterization of the Heavy Metals Contaminating the River Nile at El-Giza Governorate, Egypt and Their Relative Bioaccumulations in Tilapia nilotica. Toxicol. Res 2008, 24, 297-305.

35. Kelter, P. B.; Grundman, J.; Hage, D. S.; Carr, J. D.; Castro-Acuña, C. M. A Discussion of Water Pollution in the United States and Mexico; with High School Laboratory Activities for the Analysis of Lead, Atrazine, and Nitrate. J. Chem. Educ. 1997, 74 (12), 1413-1421.

36. Sun, D.; Cao, R.; Bi, W.; Weng, J.; Hong, M.; Liang, Y. Syntheses and Characterizations of a Series of Silver-Carboxylate Polymers. Inorg. Chim. Acta 2004, 357 (4), 991-1001.

37. Luan, Y.; Qi, Y.; Jin, Z.; Peng, X.; Gao, H.; Wang, G. Synthesis of a Flower-Like ZrBased Metal-Organic Framework and Study of its Catalytic Performance in the Mannich Reaction. RSC Adv 2015, 5 (25), 19273-19278.

38. Téllez S, C. A.; Hollauer, E.; Mondragon, M. A.; Castaño, V. M. Fourier Transform Infrared and Raman Spectra, Vibrational Assignment and ab Initio Calculations of Terephthalic Acid and Related Compounds. Spectroc Acta A 2001, 57 (5), 993-1007.

39. do Nascimento, J. F. S.; Barros, B. S.; Kulesza, J.; de Oliveira, J. B. L.; Pereira Leite, A. K.; de Oliveira, R. S. Influence of Synthesis Time on the Microstructure and Photophysical Properties of Gd-MOFs Doped with Eu3+. Mater. Chem. Phys. 2017, 190, 166-174.

40. Gutiérrez, M.; Martín, C.; Souza, B. E.; Van der Auweraer, M.; Hofkens, J.; Tan, J.-C. Highly Luminescent Silver-Based MOFs: Scalable Eco-Friendly Synthesis Paving the Way for Photonics Sensors and Electroluminescent Devices. Appl. Mater. Today 2020, 21, article no. 100817.

41. Li, H.; Eddaoudi, M.; O'Keeffe, M.; Yaghi, O. M. Design and Synthesis of an Exceptionally Stable and Highly Porous Metal-Organic Framework. Nature 1999, 402 (6759), 276-279.

42. Tranchemontagne, D. J.; Hunt, J. R.; Yaghi, O. M. Room Temperature Synthesis of MetalOrganic Frameworks: MOF-5, MOF-74, MOF-177, MOF-199, and IRMOF-0. Tetrahedron 2008, 64 (36), 8553-8557.

43. Möslein, A. F.; Gutiérrez, M.; Cohen, B.; Tan, J.-C. Near-Field Infrared Nanospectroscopy Reveals Guest Confinement in Metal-Organic Framework Single Crystals. Nano Letters 2020, 20 (10), 7446-7454.

44. Kim ID., C. S., Kim SJ., Jang JS., Exhaled Breath Sensors. In Smart Sensors for Health and Environment Monitoring, KAIST Research Series. Springer, Dordrecht: 2015.

45. Miekisch, W.; Schubert, J. K. From Highly Sophisticated Analytical Techniques to LifeSaving Diagnostics: Technical Developments in Breath Analysis. TRAC-Trend Anal. Chem. 2006, 25 (7), 665-673.

46. Ma, Y.; Tang, H.; Dong, X.; Wang, K.; Liu, M.; Wang, Y. A Dual-Functional 3D Coordination Polymer as a Luminescent Sensor for Acetone in an Aqueous Medium and Detecting the Temperature. Inorg. Chem. Commun. 2019, 105, 13-19.

47. Sotelo-Gonzalez, E.; Coto-Garcia, A. M.; Fernandez-Argüelles, M. T.; Costa-Fernandez, J. M.; Sanz-Medel, A. Immobilization of Phosphorescent Quantum Dots in a Sol-Gel Matrix for Acetone Sensing. Sens. Actuators B: Chem. 2012, 174, 102-108. 
48. Zhang, Q.; Wang, J.; Kirillov, A. M.; Dou, W.; Xu, C.; Xu, C.; Yang, L.; Fang, R.; Liu, W. Multifunctional Ln-MOF Luminescent Probe for Efficient Sensing of Fe3+, Ce3+, and Acetone. ACS Appl. Mater. Interfaces 2018, 10 (28), 23976-23986.

49. Peedikakkal, A. M. P.; Qamar, M. Emissive Lead(II) Benzenedicarboxylate Metal-Organic Frameworks. J. Chem. Sci. 2018, 130 (5), article no. 45.

50. Li, Z.-P.; Xing, Y.-H.; Wang, C.-G.; Li, J.; Zeng, X.-Q.; Ge, M.-F.; Niu, S.-Y. A New Coordination Polymer [Pb(1,4-BDC)]n Containing a Unique $\mu 6$-Bridging Coordination Mode. $Z$. Anorg. Allg. Chem 2009, 635 (11), 1650-1653.

51. Tan, Y.-X.; Meng, F.-Y.; Wu, M.-C.; Zeng, M.-H. Two Pb(II) Dicarboxylates Constructed by Rigid Terephthalate or Flexible $\mathrm{d}(+)$-Camphorate with Different 3D Motif Based on Cooperative Effect of Steric Hindrance of Ligand and Lone Pair Electrons. J. Mol. Struct. 2009, $928(1), 176-181$.

52. Zhang, L.; Li, Z.-J.; Lin, Q.-P.; Qin, Y.-Y.; Zhang, J.; Yin, P.-X.; Cheng, J.-K.; Yao, Y.-G. Synthesis, Structure, and Luminescent Properties of Hybrid Inorganic-Organic Framework Materials Formed by Lead Aromatic Carboxylates: Inorganic Connectivity Variation from 0D to 3D. Inorg. Chem. 2009, 48 (14), 6517-6525.

53. Dale, S. H., Elsegood, M. R. J., Kainth, S. Poly-[lead(II)- $\mu 2$-aqua- $\mu 4$-tereph-thalato]. Acta Crystallogr. Sect. C 2004, 60, 76-78.

54. Elrasheedy, A.; Nady, N.; Bassyouni, M.; El-Shazly, A. Metal Organic Framework Based Polymer Mixed Matrix Membranes: Review on Applications in Water Purification. Membranes (Basel) 2019, 9 (7), article no. 88.

55. Li, X.; Liu, Y.; Wang, J.; Gascon, J.; Li, J.; Van der Bruggen, B. Metal-Organic Frameworks Based Membranes for Liquid Separation. Chem. Soc. Rev. 2017, 46 (23), 7124-7144.

56. Liu, G.; Chernikova, V.; Liu, Y.; Zhang, K.; Belmabkhout, Y.; Shekhah, O.; Zhang, C.; Yi, S.; Eddaoudi, M.; Koros, W. J. Mixed Matrix Formulations with MOF Molecular Sieving for Key Energy-Intensive Separations. Nat. Mater. 2018, 17 (3), 283-289.

57. Seoane, B.; Coronas, J.; Gascon, I.; Benavides, M. E.; Karvan, O.; Caro, J.; Kapteijn, F.; Gascon, J. Metal-Organic Framework Based Mixed Matrix Membranes: a Solution for Highly Efficient CO2 Capture? Chem. Soc. Rev. 2015, 44 (8), 2421-2454.

58. Tanh Jeazet, H. B.; Staudt, C.; Janiak, C. Metal-Organic Frameworks in Mixed-Matrix Membranes for Gas Separation. Dalton Trans 2012, 41 (46), 14003-14027.

59. Mahdi, E. M.; Tan, J.-C. Dynamic Molecular Interactions Between Polyurethane and ZIF8 in a Polymer-MOF Nanocomposite: Microstructural, Thermo-Mechanical and Viscoelastic effects. Polymer 2016, 97, 31-43.

60. Flyagina, I. S.; Mahdi, E. M.; Titov, K.; Tan, J.-C. Thermo-Mechanical Properties of Mixed-Matrix Membranes Encompassing Zeolitic Imidazolate Framework-90 and Polyvinylidine Difluoride: ZIF-90/PVDF Nanocomposites. APL Mater 2017, 5 (8), article no. 086104.

61. Gutiérrez, M.; López-González, M.; Sánchez, F.; Douhal, A. Efficient Light Harvesting within a C153@Zr-based MOF Embedded in a Polymeric Film: Spectral and Dynamical Characterization. Phys. Chem. Chem. Phys. 2017, 19 (27), 17544-17552.

62. Mahdi, E. M.; Chaudhuri, A. K.; Tan, J.-C. Capture and Immobilisation of Iodine (I2) Utilising Polymer-Based ZIF-8 Nanocomposite Membranes. Mol. Sys. Des. Eng. 2016, 1 (1), 122131.

63. Moreton, J. C.; Denny, M. S.; Cohen, S. M. High MOF Loading in Mixed-Matrix Membranes Utilizing Styrene/Butadiene Copolymers. Chem. Commun. 2016, 52 (100), 1437614379 . 
64. Souza, B. E.; Donà, L.; Titov, K.; Bruzzese, P.; Zeng, Z.; Zhang, Y.; Babal, A. S.; Möslein, A. F.; Frogley, M. D.; Wolna, M.; Cinque, G.; Civalleri, B.; Tan, J.-C. Elucidating the Drug Release from Metal-Organic Framework Nanocomposites via In Situ Synchrotron Microspectroscopy and Theoretical Modeling. ACS Appl. Mater. Interfaces 2020, 12 (4), 51475156. 


\section{Supporting Information \\ for}

Facile and Fast Transformation of Non-Luminescent to

Highly Luminescent MOFs: Acetone Sensing for Diabetes

Diagnosis and Lead Capture from Polluted Water

Mario Gutiérrez, ${ }^{1}$ Annika F. Möslein, ${ }^{1}$ and Jin-Chong Tan ${ }^{1 *}$

${ }^{1}$ Multifunctional Materials \& Composites (MMC) Laboratory, Department of Engineering Science, University of Oxford, Parks Road, Oxford OX1 3PJ, United Kingdom.

*Corresponding author: jin-chong.tan@eng.ox.ac.uk 
Figure S1. FESEM images of different regions found in OX-1. A) Shows microsized OX-1 crystals while B) illustrates the small nanoplates.
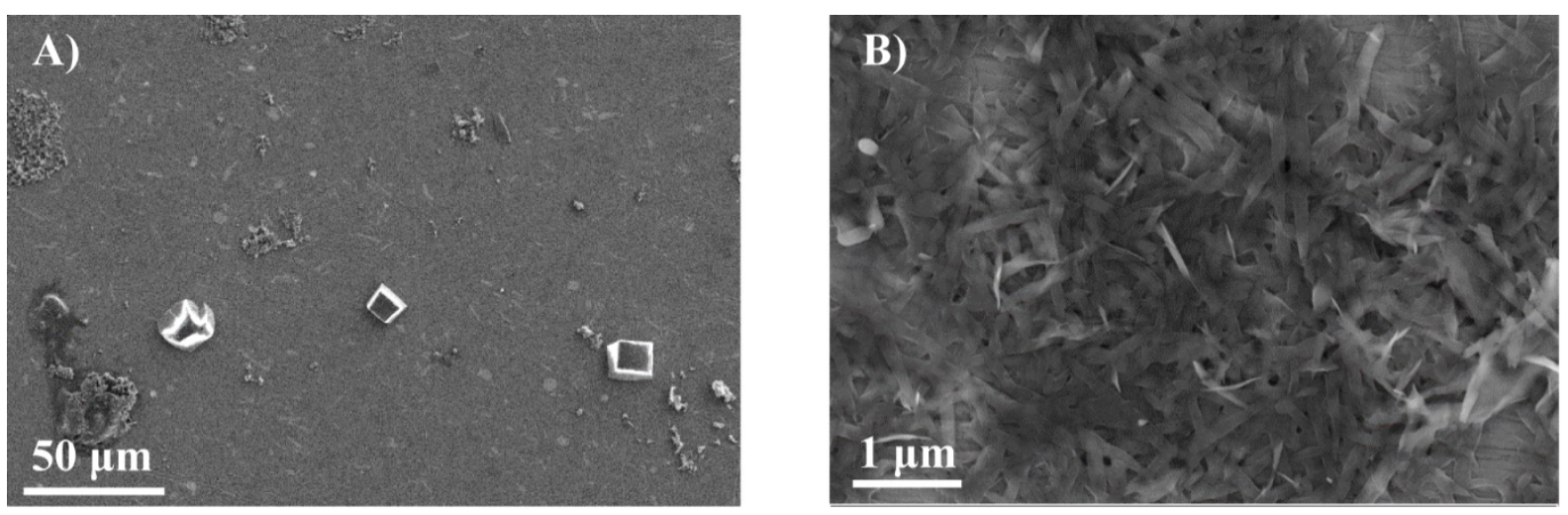

Figure S2. FESEM-EDX images of Ag/OX-1 5min showing the concurrent presence of OX-1 big crystals and small transformed OX-2 ones.
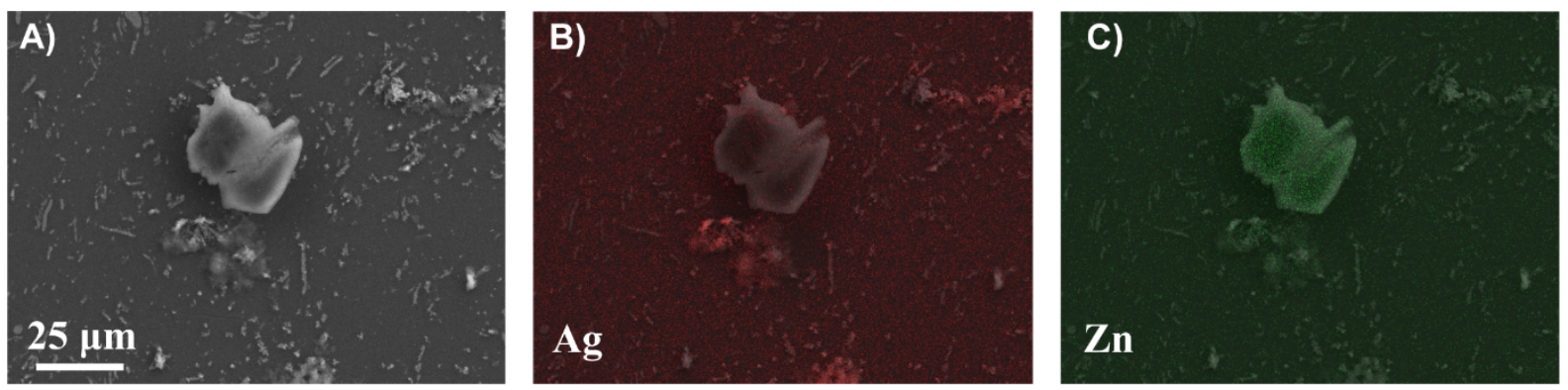

Figure S3. FESEM-EDX images of Ag/OX-1 1 h showing the coexistence of big crystals of OX-1 and nanocrystals of transformed OX-2.
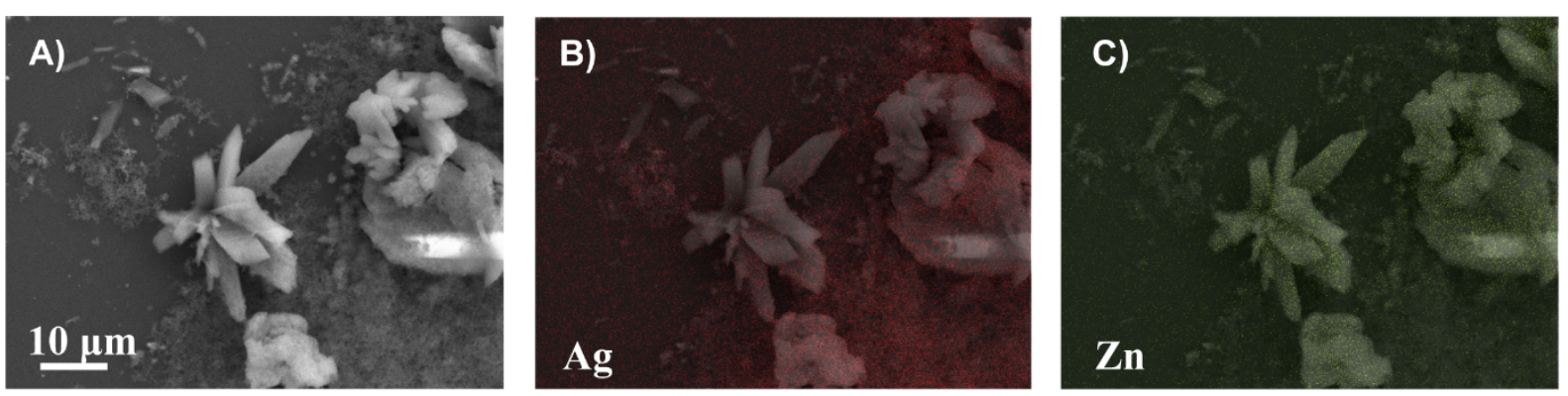
Figure S4. FESEM-EDX images of a large area found in Ag/OX-1 24h, where microsized OX-1 crystals as well as converted OX-2 nanocrystals are observed.
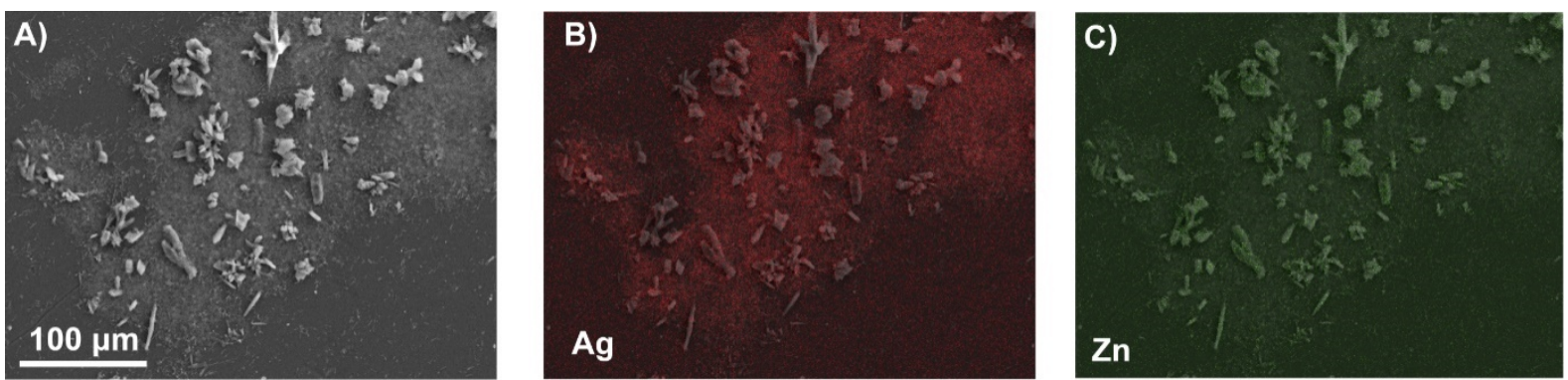
Figure S5. Excitation-emission maps of A) Ag/OX-1 5min and B) Ag/OX-1 24h. The inset shows the emission quantum yield of each sample upon excitation at $330 \mathrm{~nm}$.

A)

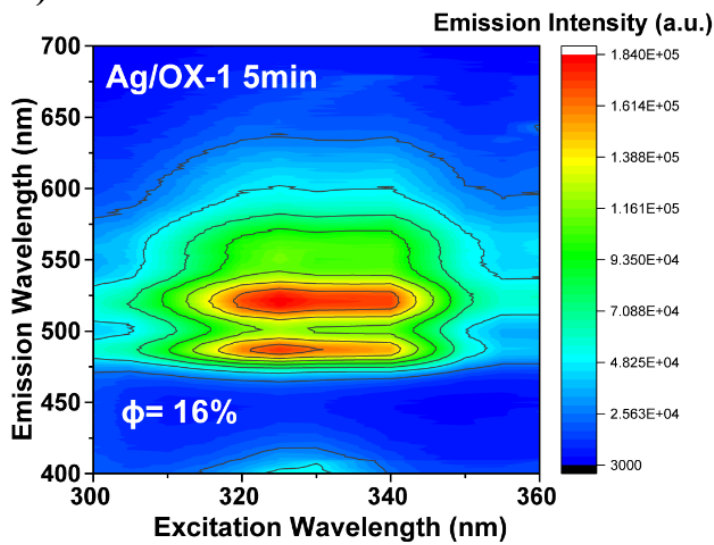

B)

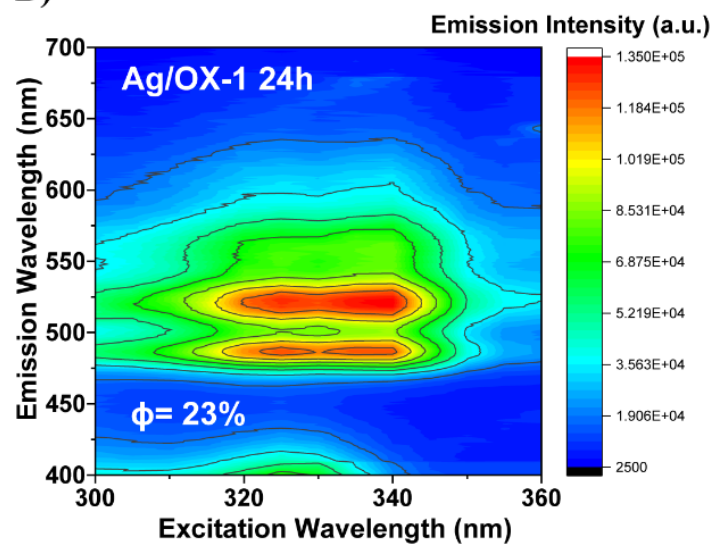


Figure S6. A-E) FESEM-EDX images of different regions found in OX-1/OX-2 mixture obtained through the one-pot synthesis method. F) PXRD patterns of pristine OX-1 and OX-2 MOF and the related OX-1/OX-2 mixture. G) Excitation-emission map of the OX1/OX-2 mixture. The inset illustrates the emission quantum yield upon excitation at 330 $\mathrm{nm}$. H) Emission spectra of the OX-1/OX-2 mixture in different solvents, and I) is a graphical representation of its emission intensity maximum in each solvent. The samples were excited at $330 \mathrm{~nm}$.
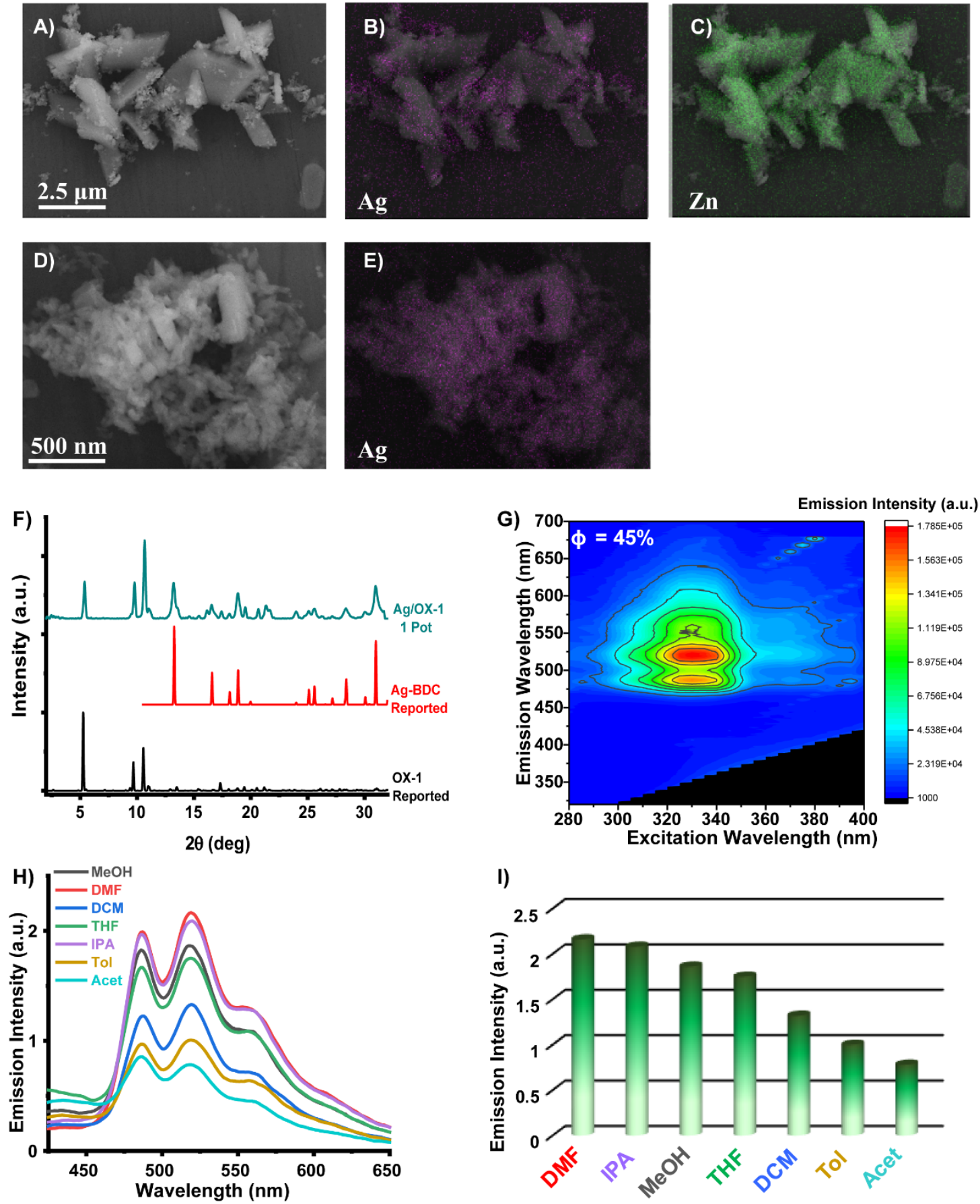
Figure S7. A) PXRD patterns and B) FTIR spectra of pristine OX-1, OX-1 treated with water and the converted $\mathrm{Ag} / \mathrm{OX}-1$ materials using different amounts of deionized water.

A)

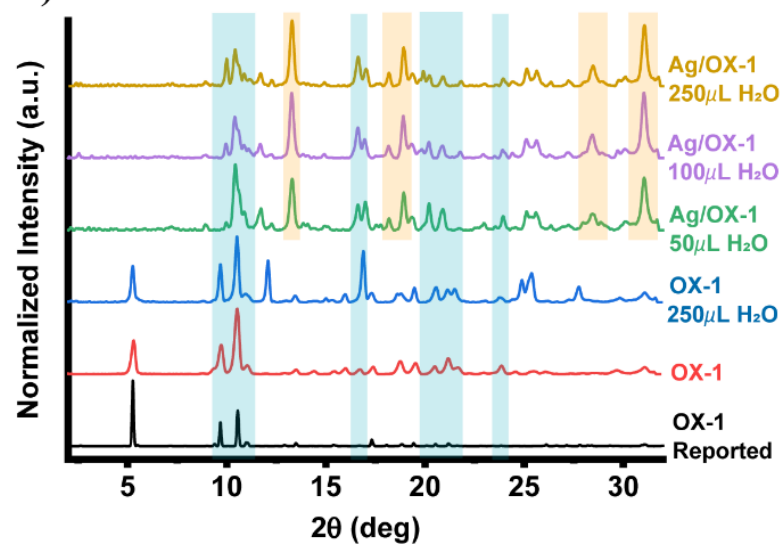

B)

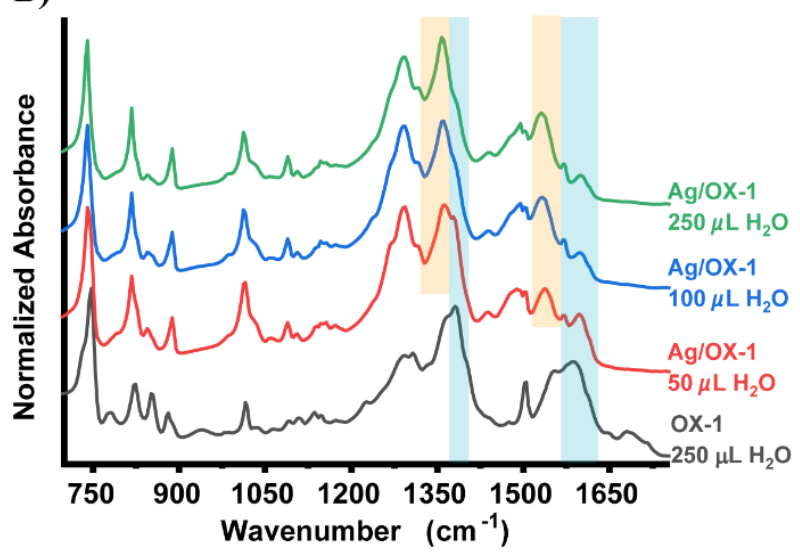


Figure S8. A) PXRD patterns and B) FTIR spectra of pristine OX-1, OX-1 treated with 1 drop of water and the mechanochemically converted Ag/OX-1. C) Photo of the OX-1 material before and after its conversion to OX-2 (grinding process) under UV irradiation. D) Photo of a mixture of $\mathrm{AgNO}_{3}$ and $\mathrm{OX}-1$ powders under UV irradiation and upon the addition of 1 drop of water and their subsequent grinding for 2 minutes.
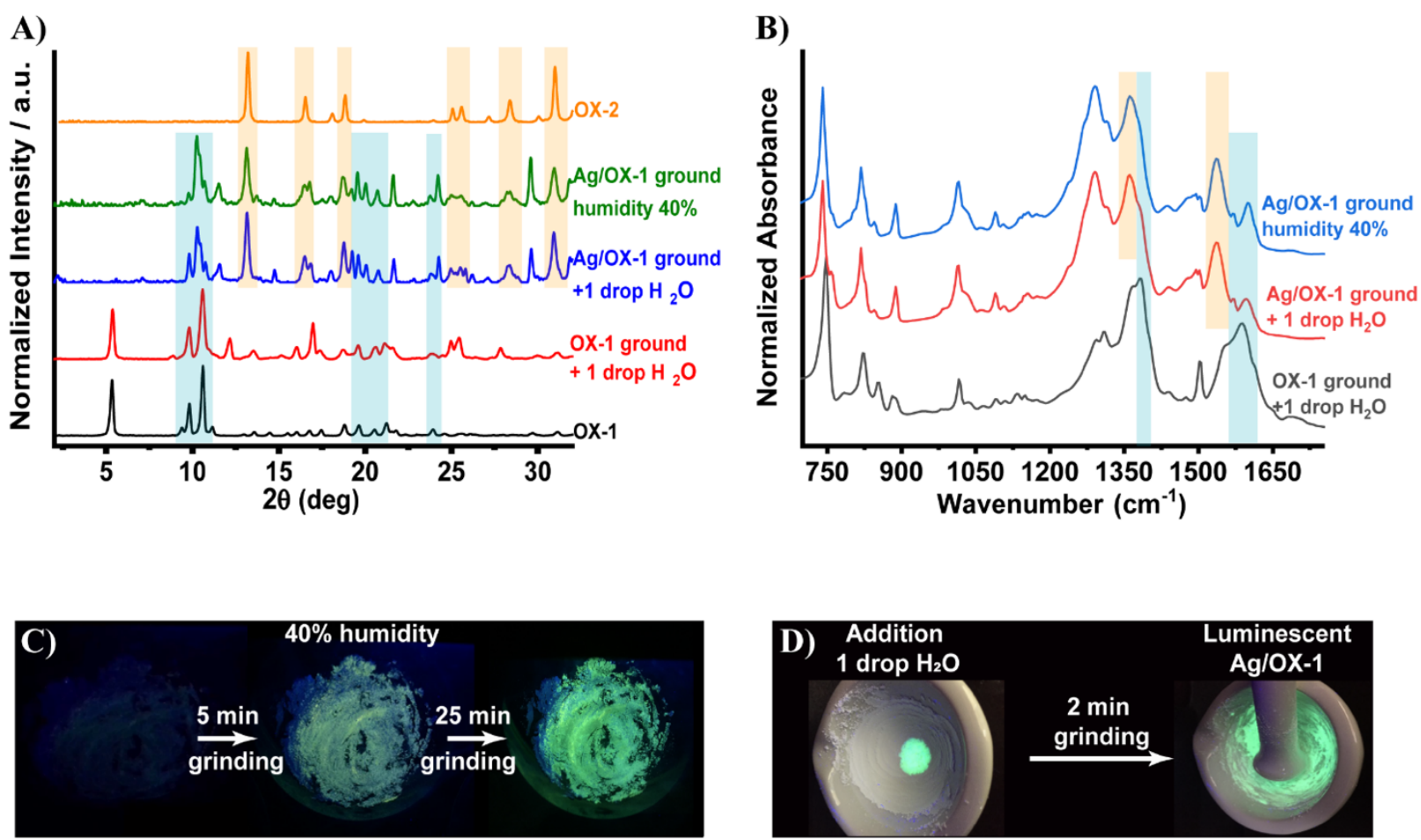
Figure S9. Excitation-emission map of A) Ag/OX-1 $50 \mu \mathrm{L} \mathrm{H}_{2} \mathrm{O}$, B) $\mathrm{Ag} / \mathrm{OX}-1100 \mu \mathrm{L} \mathrm{H}_{2} \mathrm{O}$ and C) $\mathrm{Ag} / \mathrm{OX}-1250 \mu \mathrm{L} \mathrm{H} \mathrm{H}_{2} \mathrm{O}$ materials. The inset shows a photo of the powders under $\mathrm{UV}$ irradiation and the emission quantum yield value of each sample upon excitation at $330 \mathrm{~nm}$. D) Emission spectra of $\mathrm{Ag} / \mathrm{OX}-1250 \mu \mathrm{L} \mathrm{H}_{2} \mathrm{O}$ in different solvents. The samples were excited at $330 \mathrm{~nm}$.
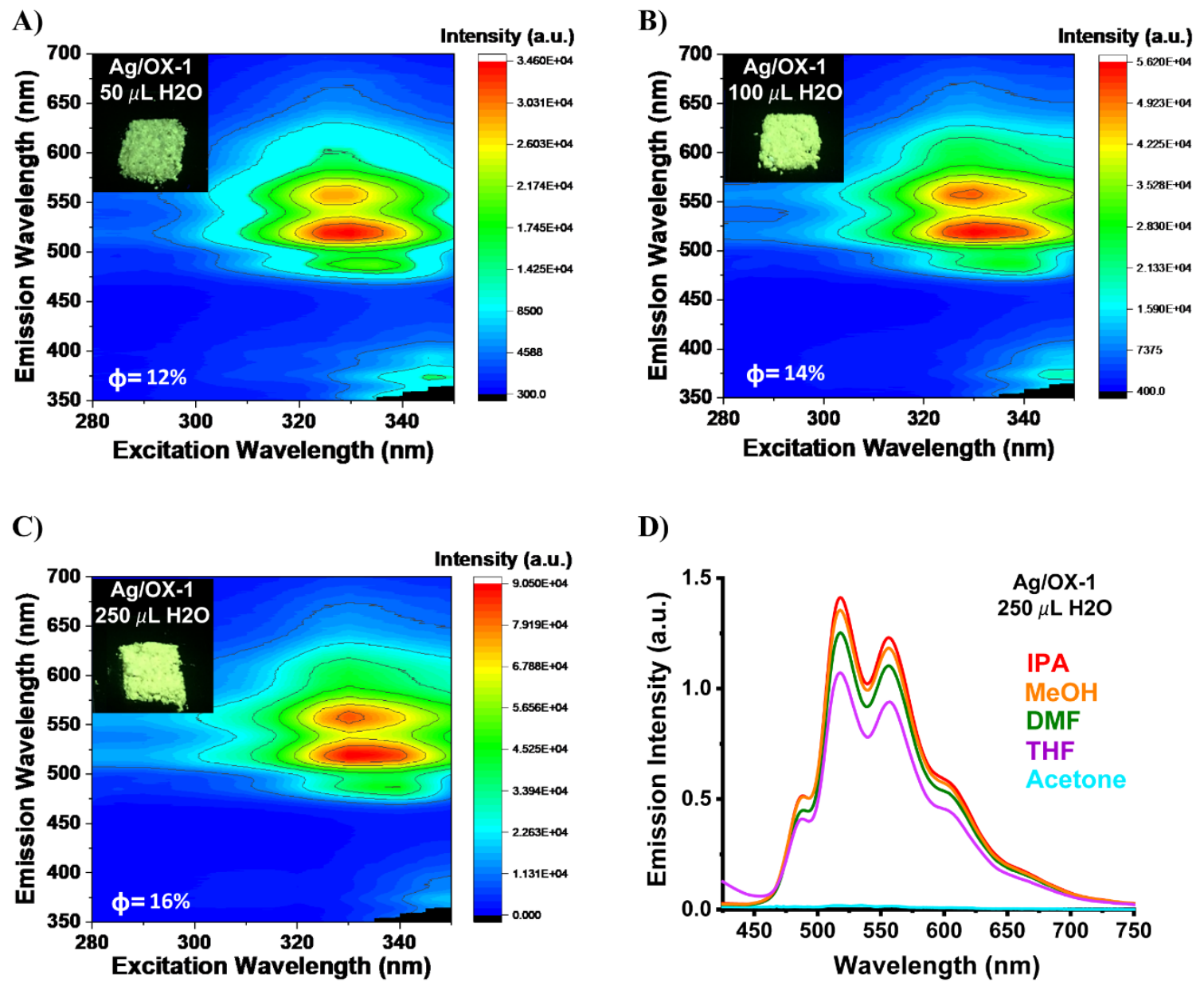
Figure S10. Excitation-emission map of A) Ag/OX-1 ground at ambient conditions and B) $\mathrm{Ag} / \mathrm{OX}-1$ ground after the addition of 1 drop of water. The inset displays the emission quantum yield of each sample upon excitation at $330 \mathrm{~nm}$. C) Emission spectra of $\mathrm{Ag} / \mathrm{OX}-1$ ground with 1 drop of water in different solvents and D) is a graphical representation of its intensity maximum in each solvent. The samples were excited at 330 nm.
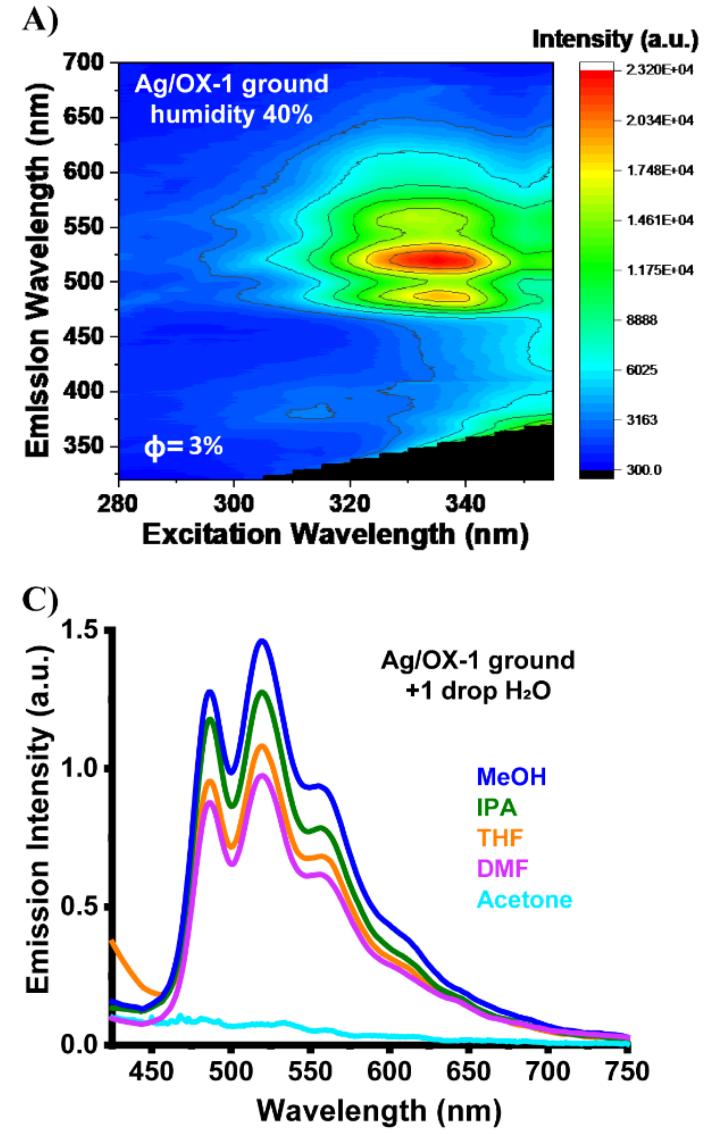

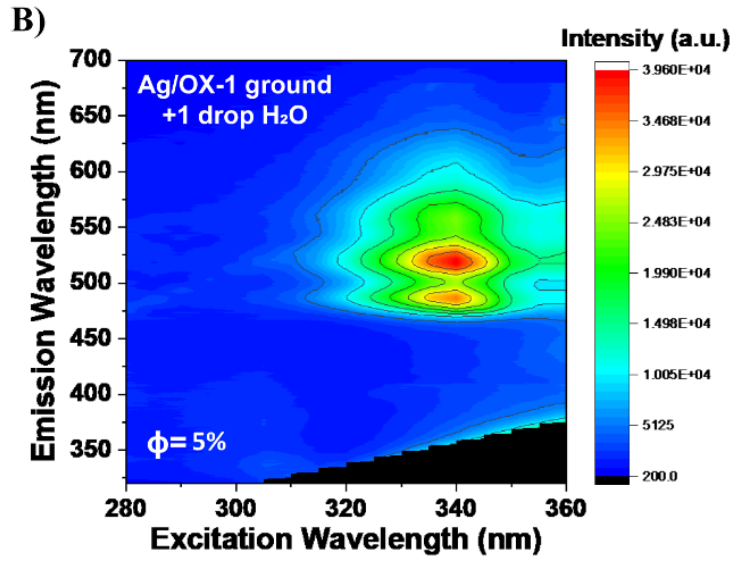

D)

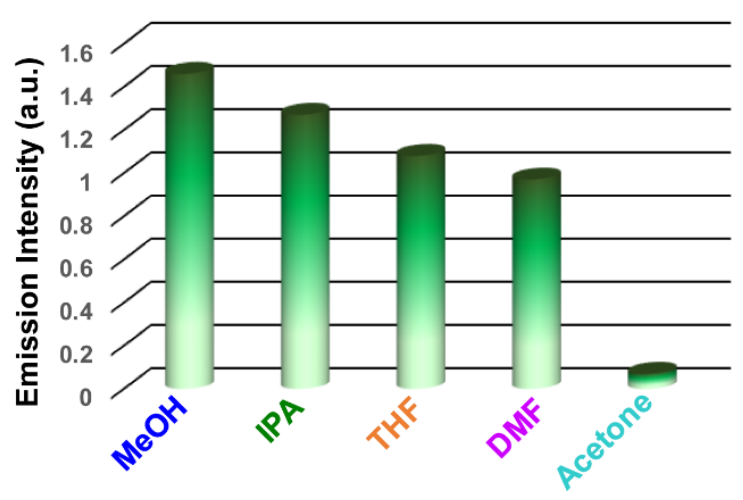


Figure S11. PXRD patterns of the OX-1/PU membrane before and after being immersed in water for 20 minutes.

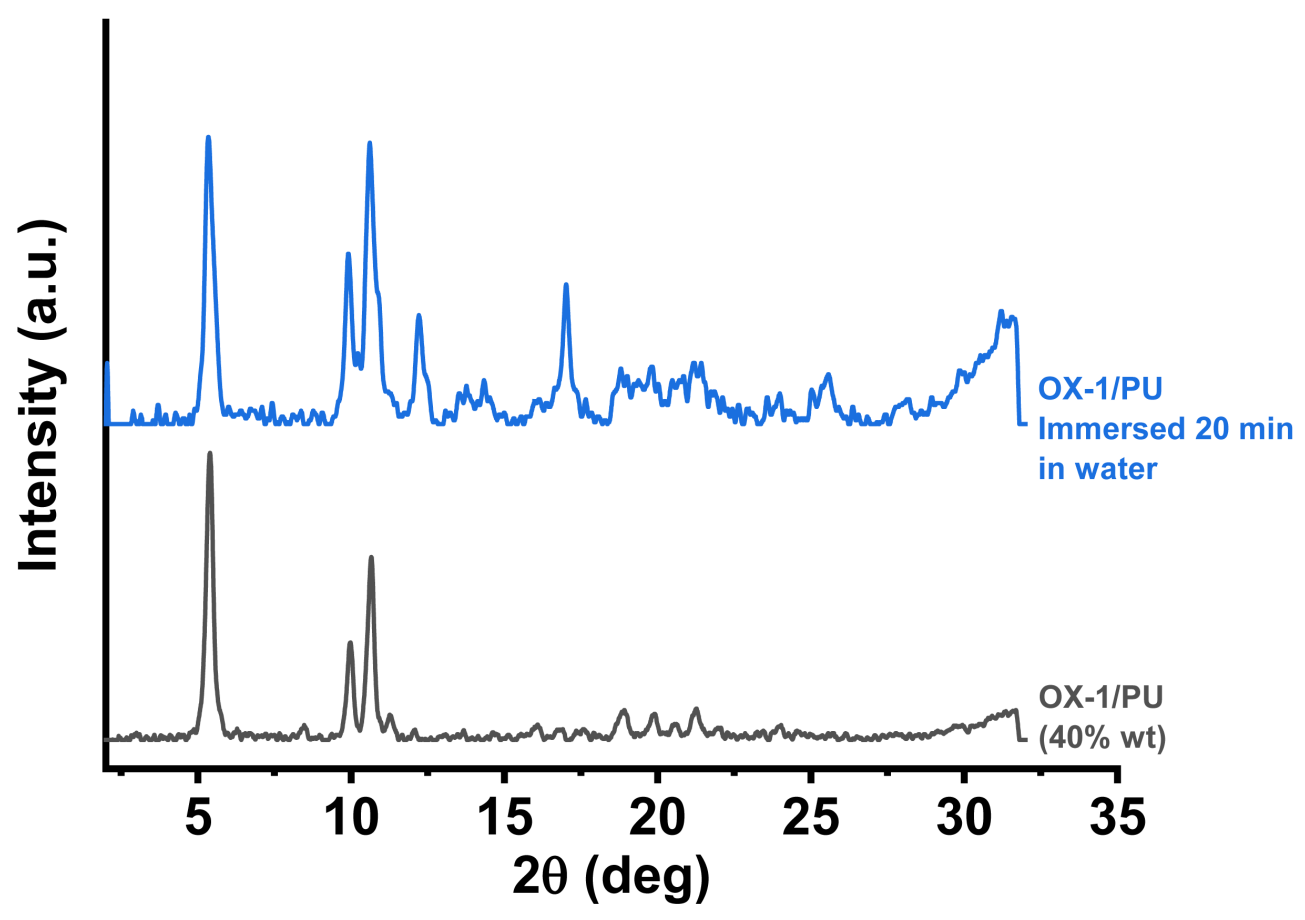

